\title{
Relating dust, gas, and the rate of star formation in M 31
}

\author{
F. S. Tabatabaei and E. M. Berkhuijsen
}

\author{
Max-Planck Institut für Radioastronomie, Auf dem Hügel 69, 53121 Bonn, Germany \\ e-mail: tabataba@mpifr-bonn.mpg.de
}

Received 3 November 2009 / Accepted 26 April 2010

\section{ABSTRACT}

\begin{abstract}
Aims. We investigate the relationships between dust and gas, and study the star formation law in M 31 .
Methods. We have derived distributions of dust temperature and dust opacity across M 31 at 45" resolution using the Spitzer data. With the opacity map and a standard dust model we de-reddened the $\mathrm{H} \alpha$ emission yielding the first $\mathrm{H} \alpha$ map of M 31 corrected for extinction. We compared the emissions from dust, $\mathrm{H} \alpha, \mathrm{HI}$, and $\mathrm{H}_{2}$ by means of radial distributions, pixel-to-pixel correlations, and wavelet cross-correlations. We calculated the star formation rate and star formation efficiency from the de-reddened $\mathrm{H} \alpha$ emission.

Results. The dust temperature steeply decreases from $30 \mathrm{~K}$ near the center to $15 \mathrm{~K}$ at large radii. The mean dust optical depth at the $\mathrm{H} \alpha$ wavelength along the line of sight is about 0.7. The radial decrease in the dust-to-gas ratio is similar to that of the oxygen abundance. Extinction is nearly linearly correlated with the total gas surface density within limited radial intervals. On scales $<2 \mathrm{kpc}$, cold dust emission is best correlated with that of neutral gas, and warm dust emission with that of ionized gas. The $\mathrm{H} \alpha$ emission is slightly better correlated with emission at $70 \mu \mathrm{m}$ than at $24 \mu \mathrm{m}$. The star formation rate in M 31 is low. In the area $6 \mathrm{kpc}<R<17 \mathrm{kpc}$, the total SFR is $\simeq 0.3 M_{\odot} \mathrm{yr}^{-1}$. A linear relationship exists between surface densities of SFR and $\mathrm{H}_{2}$. The Kennicutt-Schmidt law between SFR and total gas has a power-law index of $1.30 \pm 0.05$ in the radial range of $R=7-11 \mathrm{kpc}$ increasing by about $0.3 \mathrm{for} R=11-13 \mathrm{kpc}$. Conclusions. The better $70 \mu \mathrm{m}-\mathrm{H} \alpha$ than $24 \mu \mathrm{m}-\mathrm{H} \alpha$ correlation plus an excess in the $24 \mu \mathrm{m} / 70 \mu \mathrm{m}$ intensity ratio indicates that other sources than dust grains, e.g. those of stellar origin, contribute to the $24 \mu \mathrm{m}$ emission. The lack of $\mathrm{H}_{2}$ in the central region could be related to the lack of HI and the low opacity/high temperature of the dust. Since neither SFR nor SFE is well correlated with the surface density of $\mathrm{H}_{2}$ or total gas, other factors than gas density must play an important role in the formation of massive stars in M 31 . The molecular depletion time scale of $1.1 \mathrm{Gyr}$ indicates that M 31 is about three times less efficient in forming young massive stars than M 33.
\end{abstract}

Key words. galaxies: individual: M 31 - galaxies: ISM - dust, extinction - ISM: general - stars: formation

\section{Introduction}

Dust, neutral gas, and ionized gas are the major components of the interstellar medium (ISM) in galaxies. Observations of their properties and inter-relationships can give strong clues to the physics governing star formation. Relationships between components in the ISM are to be expected. Observations have shown that in the Galaxy dust and neutral gas are well mixed. In dense clouds of molecular gas mixed with cold dust most of the stars are formed. They subsequently heat the dust and gas in their surroundings and ionize the atomic gas. As the major coolants of the ISM are continuum emission and line emission at various frequencies, a close comparison of these emissions could shed light on spatial and physical connections between the emitting components. Present-day IR and radio telescopes have produced sensitive high-resolution maps of several nearby galaxies, which are ideal laboratories for studying the interplay between the ISM and star formation (e.g. Kennicutt et al. 2007; Bigiel et al. 2008; Verley et al. 2009).

The spiral galaxy nearest to us, the Andromeda Nebula (NGC 224), is a highly inclined Sb galaxy of low surface brightness. Table 1 lists the positional data on M 31. Its proximity and large extent on the sky $\left(>5^{\circ} \times 1^{\circ}\right)$ enable detailed studies of the ISM over a wide radial range.

Surveys of M 31 at high angular resolution $\left(<1^{\prime}\right)$ are available at many wavelengths. In the HI line the galaxy was mapped by Brinks \& Shane (1984) at $24^{\prime \prime} \times 36^{\prime \prime}$ resolution, the northeastern half by Braun (1990) at 10" resolution, and most recently,
Table 1. Positional data adopted for M 31.

\begin{tabular}{ll}
\hline \hline Position of nucleus & $\mathrm{RA}=00^{\mathrm{h}} 42^{\mathrm{m}} 45.97^{\mathrm{s}}$ \\
$(\mathrm{J} 2000)$ & $\mathrm{Dec}=41^{\circ} 16^{\prime} 11.64^{\prime \prime}$ \\
Position angle of major axis & $37^{\circ}$ \\
Inclination $^{1}$ & $75^{\circ}\left(0^{\circ}=\right.$ face on $)$ \\
Distance $^{2}$ & $780 \pm 40 \mathrm{kpc}^{3}$ \\
\hline
\end{tabular}

Notes. ${ }^{(1)}$ Berkhuijsen (1977) and Braun (1991). ${ }^{(2)}$ Stanek \& Garnavich (1998) . (3) $1^{\prime}=227 \pm 12 \mathrm{pc}$ along major axis.

the entire galaxy with high sensitivity by Braun et al. (2009) at a resolution of $15^{\prime \prime}$. Nieten et al. (2006) made a survey in the ${ }^{12} \mathrm{CO}(1-0)$ line at a resolution of 23 ". Devereux et al. (1994) observed M 31 in the $\mathrm{H} \alpha$ line to obtain the distribution of the ionized gas. The dust emission from M 31 has recently been observed by the multiband imaging photometer Spitzer (MIPS, Rieke et al. 2004) with high sensitivity at $24 \mu \mathrm{m}, 70 \mu \mathrm{m}$, and $160 \mu \mathrm{m}$ at resolutions $\leq 40^{\prime \prime}$.

The relationships between gas and dust, as well as between gas and star formation, in M 31 have been studied in the past at resolutions of several arcminutes. Walterbos \& Schwering (1987) derived a nearly constant dust temperature across M 31 using the IRAS $100 \mu \mathrm{m}$ and $60 \mu \mathrm{m}$ maps. They also found a strong increase in the atomic gas-to-dust surface density ratio with increasing radius. This increase was confirmed by Walterbos \& Kennicutt (1988) who used optical extinction as 
Table 2. M 31 data used in this study.

\begin{tabular}{llll}
\hline \hline Wavelength & Resolution & Telescope & Ref. \\
\hline $160 \mu \mathrm{m}$ & $40^{\prime \prime}$ & Spitzer & Gordon et al. (2006) \\
$70 \mu \mathrm{m}$ & $18^{\prime \prime}$ & Spitzer & Gordon et al. (2006) \\
$24 \mu \mathrm{m}$ & $6^{\prime \prime}$ & Spitzer & Gordon et al. (2006) \\
$2.6 \mathrm{~mm}{ }^{12} \mathrm{CO}(1-0)$ & $23^{\prime \prime}$ & IRAM 30-m & Nieten et al. (2006) \\
$21 \mathrm{~cm} \mathrm{HI}$ & $24^{\prime \prime} \times 30^{\prime \prime}$ & WSRT & Brinks \& Shane (1984) \\
$6570 \AA \AA \mathrm{H} \alpha$ & $2^{\prime \prime}($ pixel size) & KPNO & Devereux et al. (1994) \\
\hline
\end{tabular}

dust tracer, and by Nieten et al. (2006) using the ISO map at $175 \mu \mathrm{m}$ (Haas et al. 1998). Interestingly, the latter authors did not find a radial increase in the molecular gas-to-dust ratio.

The dependence of star formation on HI surface density in M 31 has been studied by a number of authors (Emerson 1974; Berkhuijsen 1977; Tenjes \& Haud 1991; Unwin 1980; Nakai \& Sofue 1982, 1984) using the number density of HII regions or of OB stars as star formation tracers. They obtained power-law indices between 0.5 and 2, possibly depending on the region in M 31, the star formation tracer and the angular resolution. Braun et al. (2009) plotted the star formation density derived from the brightnesses at $8 \mu \mathrm{m}, 24 \mu \mathrm{m}$, and UV against the surface densities of molecular gas, HI, and total gas, but did not fit power laws to their data.

The high-resolution data available for M 31 show the morphologies of the emission from dust and gas components in detail. We apply a 2-D wavelet analysis technique (Frick et al. 2001) to the MIPS IR data (Gordon et al. 2006) and the gas $\left(\mathrm{HI}, \mathrm{H}_{2}\right.$, and $\mathrm{H} \alpha$ ) maps to study the scale distribution of emission power and to separate the diffuse emission components from compact sources. We then compare the waveletdecomposed maps at various spatial scales. We also use pixel-topixel (Pearson) correlations to derive quantitative relations not only between different ISM components but also between them and the present-day star formation rate.

Following Walterbos \& Schwering (1987) and Haas et al. (1998), we derive the dust temperature assuming a $\lambda^{-2}$ emissivity law for the MIPS bands at which the emission from the big grains and hence the LTE condition is relevant, and present a map of the dust color temperature. We also obtain the distribution of the optical depth and analyze the gas-to-dust surfacedensity ratio at a resolution of $45^{\prime \prime}(170 \mathrm{pc} \times 660 \mathrm{pc}$ along the major and minor axis, respectively, in the galaxy plane), 9 times higher than before (Walterbos \& Schwering 1987). We use the optical depth map to de-redden the $\mathrm{H} \alpha$ emission observed by Devereux et al. (1994) yielding the distribution of the absorption-free emission from the ionized gas, and use this as an indicator of massive star formation. We compare it with the distributions of neutral gas to obtain the dependence of the star formation rate on gas surface density.

The paper is organized as follows: The relevant data sets are described in Sect. 2. In Sect. 3 we derive maps of the dust color temperature and optical depth, and correct the $\mathrm{H} \alpha$ emission for absorption by dust. Radial profiles of the dust and gas emission and of the various gas-to-dust ratios are obtained in Sect. 4. Section 5 is devoted to wavelet decompositions and wavelet spectra of the dust and gas distributions, and their cross correlations. Complementary, we discuss in Sect. 6 classical correlations between gas and dust. In Sect. 7 the dependence of the star formation rate on the gas surface density is presented. Finally, in Sect. 8 we summarize our results.

\section{Data}

Table 2 summarizes the data used in this work. M 31 was mapped in IR (at $24 \mu \mathrm{m}, 70 \mu \mathrm{m}$, and $160 \mu \mathrm{m}$ ) by MIPS in August 2004 covering a region of about $1^{\circ} \times 3^{\circ}$ (Gordon et al. 2006). The basic data reduction and mosaicing was performed using the MIPS instrument team Data Analysis Tool versions 2.90 (Gordon et al. 2005).

M 31 was observed in the ${ }^{12} \mathrm{CO}(1-0)$ line with the IRAM telescope by Nieten et al. (2006) at a resolution of $23^{\prime \prime}$. They derived the distribution of the molecular gas using a constant conversion factor of $\mathrm{X}_{\mathrm{CO}}=1.9 \times 10^{20} \mathrm{~mol} . \mathrm{K}^{-1} \mathrm{~km}^{-1} \mathrm{~s}$. The galaxy was observed in the $21-\mathrm{cm}$ HI line with the Westerbork interferometer by Brinks \& Shane (1984) at a resolution of $24^{\prime \prime} \times 36^{\prime \prime}$. The HI survey has been corrected for missing spacings. The $\mathrm{H} \alpha$ observations of Devereux et al. (1994) were carried out on the Case Western Burell-Schmidt telescope at the Kitt Peak National Observatory, providing a $2^{\circ} \times 2^{\circ}$ field of view.

Although the resolution of $40^{\prime \prime}$ of the $160 \mu \mathrm{m}$ image is the lowest of the data listed in Table 2, we smoothed all maps to a Gaussian beam with a half-power width of $45^{\prime \prime}$ for a comparison with radio continuum data at $20 \mathrm{~cm}$ (Hoernes et al. 1998) in a forthcoming study (Tabatabaei et al. in prep.). As the point spread function (PSF) of the MIPS data is not Gaussian, we convolved the MIPS images using custom kernels created with Fast Fourier transforms to account for the detailed structure of the PSFs. Details of the kernel creation can be found in Gordon et al. (2007).

After convolution, the maps were transformed to the same grid of $15^{\prime \prime}$ width with the reference coordinates and position angle of the major axis given in Table 1. Finally, they were cut to a common extent of $110^{\prime} \times 38.5$, for which most data sets are complete. The field is not centred on the nucleus of M 31, but extends to 56!25 along the northern major axis (corresponding to a radius of $R=12.8 \mathrm{kpc}$ ) and to 53.75 along the southern major axis $(R=12.2 \mathrm{kpc})$. The $\mathrm{H}_{2}$ map of Nieten et al. (2006) extends to 48.5 along the southern major axis $(R=11.0 \mathrm{kpc})$. With an extent of $19 ! 25$ along the minor axis in both directions, the field covers radii of $R<16.9 \mathrm{kpc}$ in the plane of M 31. Hence, radial profiles derived by averageing the data in circular rings in the plane of the galaxy (equivalent to elliptical rings in the plane of the sky) are incomplete at $R>12 \mathrm{kpc}$ because of missing data near the major axis.

\section{Dust temperature and opacity}

Walterbos \& Schwering (1987) extensively studied the distributions of the dust temperature and opacity in M 31 using the IRAS data at $60 \mu \mathrm{m}$ and $100 \mu \mathrm{m}$ smoothed to a resolution of $4.3 \times 6 ! 9$. Assuming a $\lambda^{-2}$ emissivity law, they found a remarkably constant dust temperature (21-22 K) across the disk between $2 \mathrm{kpc}$ and $15 \mathrm{kpc}$ radius. Using this temperature, they obtained the opacity distribution at $100 \mu \mathrm{m}$. Below we apply a similar method 


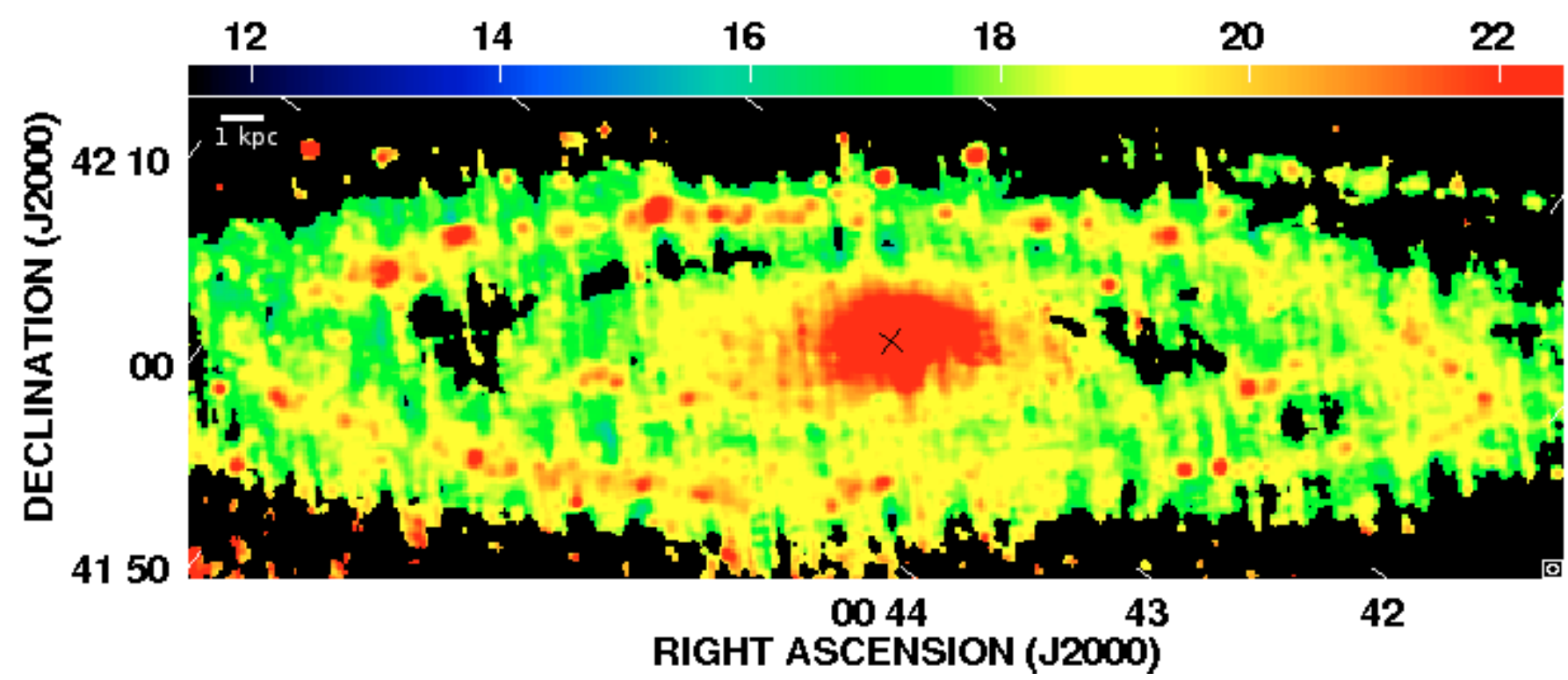

Fig. 1. Dust temperature in M 31 obtained from the ratio $I_{70} \mu \mathrm{m} / I_{160 \mu \mathrm{m}}$ based on the Spitzer MIPS data. Only pixels with intensity above $3 \times$ noise level were used. The angular resolution of $45^{\prime \prime}$ is shown in the lower right-hand corner of the map. The cross indicates the location of the center. The bar at the top gives the dust temperature in Kelvin.

to the $70 \mu \mathrm{m}$ and $160 \mu \mathrm{m}$ MIPS maps to derive the distributions of dust temperature and optical depth at the $\mathrm{H} \alpha$ wavelength at higher resolution and sensitivity.

\subsection{Dust temperature}

We derived the color temperature of the dust, $T_{\mathrm{d}}$, between $70 \mu \mathrm{m}$ and $160 \mu \mathrm{m}$ assuming a $\lambda^{-2}$ emissivity law that should be appropriate for interstellar grains emitting at these wavelengths (Andriesse 1974; Draine \& Lee 1984). The resulting dust temperature map (Fig. 1) and a histogram of the temperatures (Fig. 2a) show that $T_{\mathrm{d}}$ varies between $15.6 \pm 0.8 \mathrm{~K}$ and $30.8 \pm 0.3 \mathrm{~K}$. The mean value of $18.7 \pm 1.4 \mathrm{~K}$ (standard deviation) is lower than that obtained by Walterbos \& Schwering (1987) and close to the ISO measurements $(16 \pm 2 \mathrm{~K})$ of Haas et al. (1998). Figure 1 shows that dust of $\sim 18 \mathrm{~K}$ exists all over M 31. Warmer dust with $T_{\mathrm{d}}>20 \mathrm{~K}$ dominates in starforming regions and in an extended area around the center of the galaxy, while cooler dust dominates in interarm regions.

Figure $2 \mathrm{~b}$ shows the dust temperature averaged in rings of $0.2 \mathrm{kpc}$ width in the plane of M 31 against radius $\mathrm{R}^{1}$. On both sides of the center the dust temperature falls very fast from about $30 \mathrm{~K}$ near the nucleus to $19 \mathrm{~K}$ at $R \simeq 4.5 \mathrm{kpc}$. To the outer parts of the galaxy, it then stays within a narrow range of about $17 \mathrm{~K}-19 \mathrm{~K}$ in the north and $16 \mathrm{~K}-19 \mathrm{~K}$ in the south. This indicates different radiation characteristics between the inner $4 \mathrm{kpc}$ and beyond. In the ring of bright emission, the so called "10 kpc ring", the temperature is clearly enhanced, especially in the northern half. Thus, in contrast to the finding of Walterbos \& Schwering (1987), $T_{\mathrm{d}}$ is not constant in the range $R=2-15 \mathrm{kpc}$ but varies between $22.5 \pm 0.5 \mathrm{~K}$ and $17.2 \pm 0.7 \mathrm{~K}$.

It is interesting that the mean dust temperature obtained between $70 \mu \mathrm{m}$ and $160 \mu \mathrm{m}$ is about $3 \mathrm{~K}$ lower in M 31 than in M 33 (Tabatabaei et al. 2007b). The emission from cold dust in

Because the spiral structure is different in the northern and southern half (northeast and southwest of the minor axis, i.e. left and right of the minor axis, respectively), we present all radial profiles for each half separately.
M 31 is stronger than in M 33, which can also be inferred from the total emission spectra based on IRAS and ISO observations (see Haas et al. 1998; Hippelein et al. 2003).

\subsection{Dust opacity distribution}

The total dust optical depth along the line of sight $\tau_{160}$ was obtained from the dust intensity at $160 \mu \mathrm{m}$ and the derived temperature. Following Tabatabaei et al. (2007b), $\tau_{160}$ was converted into the dust optical depth at the wavelength of the $\mathrm{H} \alpha$ line, $\tau_{\mathrm{H} \alpha}$, by multiplying it by the ratio of the dust extinction coefficient per unit mass at the corresponding wavelengths, $\tau_{\mathrm{H} \alpha} \simeq 2200 \tau_{160}$ (see e.g. Fig. 12.8 of Krügel 2003). Figure 3 shows the distribution of $\tau_{\mathrm{H} \alpha}$ across the disk of M 31 at an angular resolution of $45^{\prime \prime}$. Regions with considerable dust opacity $\left(\tau_{\mathrm{H} \alpha}>0.6\right)$ follow the spiral arms, even the inner arms which are either weak or not detected in $\mathrm{H} \alpha$ emission. The high opacity clumps $\left(\tau_{\mathrm{H} \alpha}>1.5\right)$, however, only occur in the arms at $\simeq 5 \mathrm{kpc}$ and in the " $10 \mathrm{kpc}$ ring". On average, the optical depth is largest in the "10 kpc ring". Hence, in this ring, dust has the highest density like the atomic gas (Brinks \& Shane 1984). Figure 4a shows the histogram of $\tau_{\mathrm{H} \alpha}$, indicating a most probable value of 0.5 and a mean value of $0.7 \pm 0.4$ across the galaxy. This agrees with the value of $\tau_{\mathrm{H} \alpha}=0.5 \pm 0.4$ that follows from the mean total extinction obtained by Barmby et al. (2000) towards 314 globular clusters.

The variation of the mean dust optical depth $\tau_{\mathrm{H} \alpha}$ with galactocentric radius is shown in Fig. 4b. In the north, $\tau_{\mathrm{H} \alpha}$ peaks not only in the "10 kpc ring" (with two maxima at $R=9.9$ and $10.9 \mathrm{kpc}$ ) but also near $5 \mathrm{kpc}$ (with two maxima at $R=4.3$ and $5.9 \mathrm{kpc}$ ). Beyond $11 \mathrm{kpc} \tau_{\mathrm{H} \alpha}$ drops with an exponential scale length of $2.48 \pm 0.07 \mathrm{kpc}$ in the north and $5.06 \pm 0.22 \mathrm{kpc}$ in the south (Sect. 4, Eq. (1)).

In Fig. 5 we compare the radial variation of $\tau_{\mathrm{H} \alpha}$ for the total area with earlier determinations. The various estimates agree well given the large uncertainties. Xu \& Helou (1996) derived $\tau_{\mathrm{V}}$ from high-resolution IRAS data using a dust heating/cooling model and a sandwich configuration of dust and stars. Although 

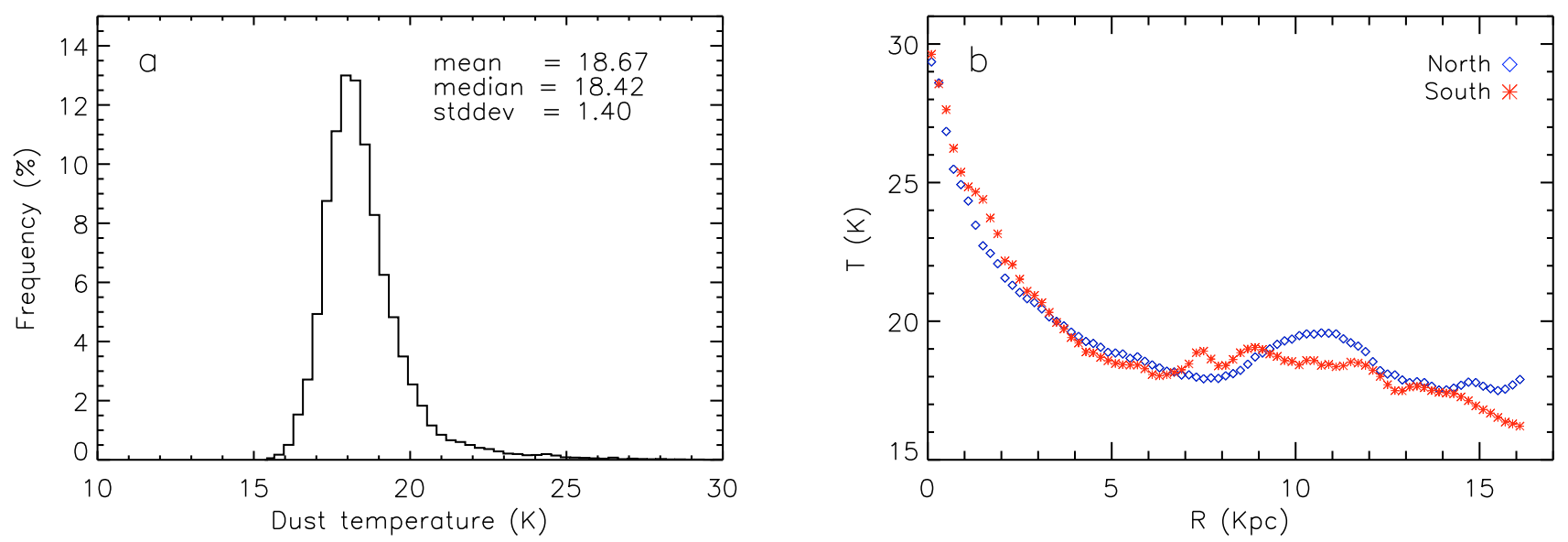

Fig. 2. a) Histogram of the dust temperature shown in Fig. 1. b) Distribution of the dust temperature in rings of $0.2 \mathrm{kpc}$ in the galactic plane in the northern and southern halves of M 31 .

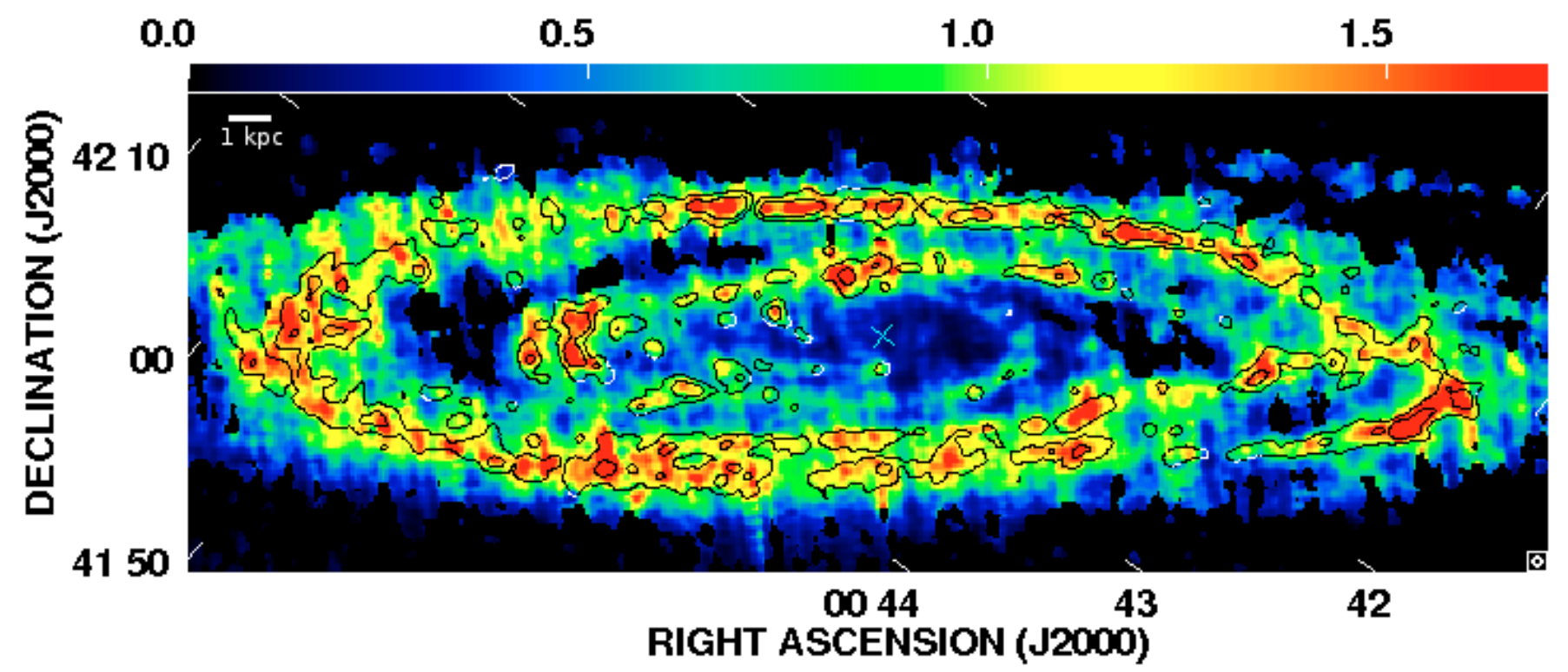

Fig. 3. Distribution of the dust optical depth at $\mathrm{H} \alpha$ wavelength in M 31. The bar at the top shows $\tau_{\mathrm{H}_{\alpha}}$. The angular resolution of 45" is shown in the lower right-hand corner of the map. The cross indicates the location of the center. Overlayed are contours of molecular gas column density $N\left(\mathrm{H}_{2}\right)$ with levels of 250 and $800 \times 10^{18} \mathrm{~mol} . \mathrm{cm}^{-2}$. Note that maxima in $\tau_{\mathrm{H}_{\alpha}}$ not always coincide with maxima in $N\left(\mathrm{H}_{2}\right)$.

they left out the discrete sources, their values may be too high because they did not include inter-arm regions in their study. Montalto et al. (2009) calculated the extinction $A_{\mathrm{FUV}}$ from the total infrared TIR-to-FUV intensity ratio and a sandwich model for stars and dust. They note that at $R<8 \mathrm{kpc}$ the geometry of M 31 may differ from the sandwich model due to the stars in the bulge, making the inner points less reliable. This would also affect the results of Xu \& Helou (1996) at $R<8 \mathrm{kpc}$. The TIR-to-FUV ratio is applicable if the dust is mainly heated by young stars, but in M 31 about 70\% of the cold dust is heated by the ISRF (Xu \& Helou 1996). Therefore, $A_{\mathrm{FUV}}$ is overestimated, as was also found for M33 (Verley et al. 2009). The curve of Tempel et al. (2010) closely agrees with our data. They derived $\tau_{\mathrm{V}}$ from MIPS data and a star-dust model. Their smooth curve underestimates $\tau_{\mathrm{V}}$ in the brightest regions by about 0.1 and may overestimate $\tau_{\mathrm{V}}$ in regions of low brightness.

The opacity map in Fig. 3 can be used to correct the $\mathrm{H} \alpha$ emission for the extinction by dust. In general, extinction depends on the relative distribution of emitting regions and dust along the line of sight and changes with the geometry (e.g. well mixed diffuse medium or shell-like in HII regions Witt \& Gordon 2000). In this study, individual HII regions are rarely resolved and the geometry is close to a mixed diffuse medium. Furthermore, there is no information about the relative position of emittors and absorbers along the line of sight. For the Milky Way, Dickinson et al. (2003) found indications of a non-uniform mixing by comparing the z-distribution of atomic gas and dust. They adopted one third of the total dust optical depth as the effective extinction as a first-order approach. This is also in agreement with Krügel (2009) taking scattering into account. Moreover, Magnier et al. (1997) found that on average the extinction comes from dust associated with only one-third of average $N(\mathrm{HI})$ in their study of OB associations along the eastern spiral arm regions of $\mathrm{M} 31$. Therefore, we use an effective optical depth $\tau_{\text {eff }}=0.33 \times \tau_{\mathrm{H} \alpha}$ in this paper. The attenuation factor for the $\mathrm{H} \alpha$ intensity then is $\mathrm{e}^{-\tau_{\text {eff }}}$ and we derive the intrinsic $\mathrm{H} \alpha$ intensity $I_{0}$ from the 

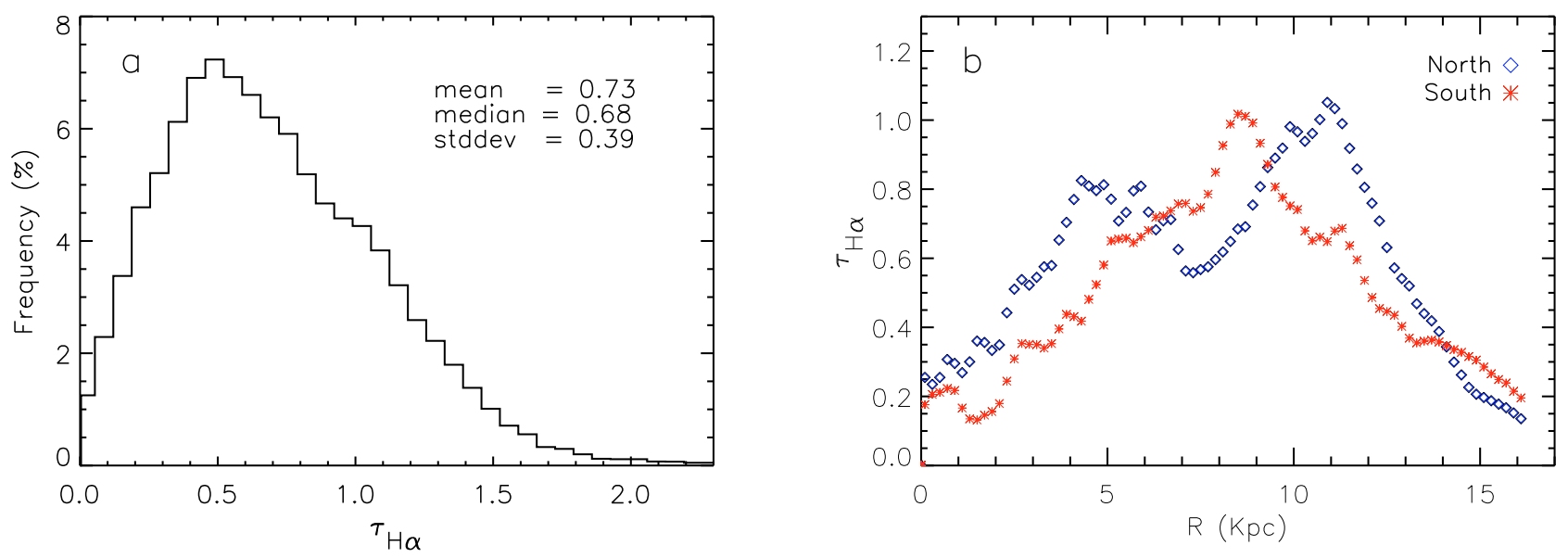

Fig. 4. a) Histogram of the dust optical depth shown in Fig. 3, b) radial distribution of the mean optical depth at the H $\alpha$ wavelength in rings of width of $0.2 \mathrm{kpc}$ in the galactic plane in the north and south of M 31 . The errors are smaller than the size of the symbols.

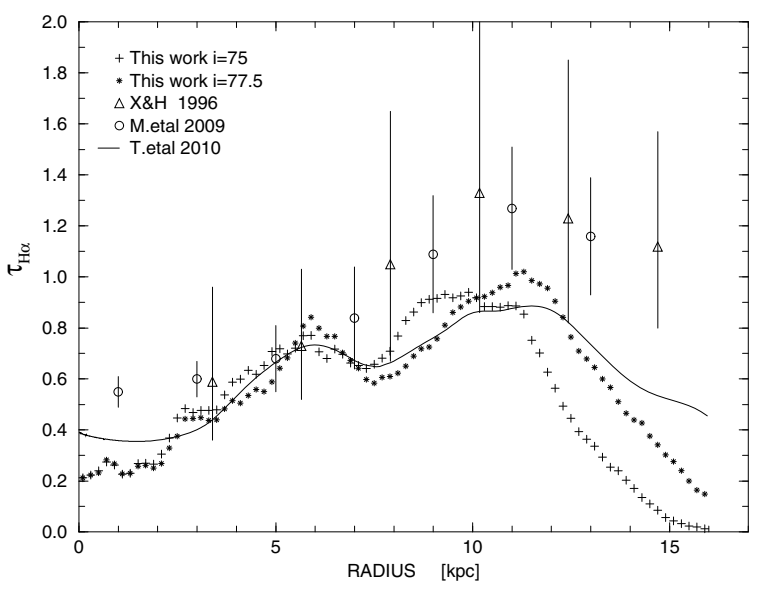

Fig. 5. Radial variation of the (total) mean optical depth in $\mathrm{H} \alpha$ along the line of sight for the full area (north+south). Plusses: our data averaged in $0.2 \mathrm{kpc}$-wide rings in the plane of M 31 using $i=75^{\circ}$; stars: same but with $i=77.5^{\circ}$ for comparison with other work; triangles: Xu \& Helou (1996), averages in $2 \mathrm{kpc}$-wide rings with $i=77^{\circ}$ (scaled to $D=780 \mathrm{kpc})$; circles: Montalto et al. (2009), averages in $2 \mathrm{kpc}$-wide rings with $i=77.6^{\circ}$; solid line: Tempel et al. (2010), semi-major axis cut through model with $i=77.5^{\circ}$. The errors in our data and in the curve of Tempel et al. (2010) are about $10 \%$ of the mean values.

observed $\mathrm{H} \alpha$ intensity $I=I_{0} \mathrm{e}^{-\tau_{\text {eff }}}$. Integration of the $\mathrm{H} \alpha$ map out to a radius of $16 \mathrm{kpc}$ yields a ratio of corrected-to-observed total $\mathrm{H} \alpha$ flux density of 1.29 , thus about $30 \%$ of the total $\mathrm{H} \alpha$ emission is obscured by dust within M 31. The corrected $\mathrm{H} \alpha$ map is shown in Fig. 10a.

Near the center $(R<1 \mathrm{kpc}), \tau_{\text {eff }}$ varies between 0.03 and 0.13 , corresponding to an extinction of $A(\mathrm{H} \alpha)=1.086 \times$ $\tau_{\text {eff }} \simeq 0.03-0.14 \mathrm{mag}$. At larger radii, the mean extinction increases, particularly in dense clouds and starforming regions, reaching a maximum of $A(\mathrm{H} \alpha) \simeq 1.2 \mathrm{mag}$ at the densest dust cloud in the south-east of the "10 kpc ring" $\left(\mathrm{RA}=00^{\mathrm{h}} 41^{\mathrm{m}}\right.$ $05.10^{\mathrm{s}}$ and $\left.\mathrm{Dec}=+40^{\circ} 38^{\prime} 17.73^{\prime \prime}\right)$. The range of extinction values agrees with that derived from the optical study of dust lanes by Walterbos \& Kennicutt (1988) and the photometric study of Williams (2003).

\section{Radial distributions of dust and gas emission}

\subsection{Radial profiles}

In this section, we present the mean surface brightness along the line of sight of dust and gas components as a function of galactocentric radius $R$. The surface brightnesses are averaged in $200 \mathrm{pc}$-wide circular rings about the nucleus in the plane of M 31. This is equivalent to averaging in elliptical rings of 53" width in the plane of the sky.

For simplicity we used a constant inclination angle of $75^{\circ}$ at all radii, appropriate for the emission at $R>30^{\prime}(6.8 \mathrm{kpc})$, although in $\mathrm{H} \alpha$ and $\mathrm{HI}$ the inner regions are seen more faceon (Ciardullo et al. 1988; Braun 1991; Chemin et al. 2009). However, using $i=75^{\circ}$ for $R<30^{\prime}$ instead of $i=68^{\circ}$ (the area-weighted mean of the inclinations for the interval $R=$ 1.9-6.8 kpc given by Chemin et al. 2009) does not change our results. The smaller inclination shifts the radial positions of the inner arms about $0.5 \mathrm{kpc}$ inwards but the general shape of the profiles remains the same, and as all profiles change in a similar way their inter-comparison is not affected. Furthermore, the results of the classical correlations for $R<30^{\prime}$ presented in Sect. 6 are the same within the errors for $i=68^{\circ}$ and $i=75^{\circ}$.

Figure 6 shows the mean IR intensities and the gas surface densities versus the galactocentric radius $R$ for the northern and southern halves of M 31 . The radial profiles of the IR emission at $24 \mu \mathrm{m}$ and $70 \mu \mathrm{m}$ are similar. The $160 \mu \mathrm{m}$ emission, representing the colder dust emission, however, shows a generally flatter radial distribution than the $24 \mu \mathrm{m}$ and $70 \mu \mathrm{m}$ emission. In particular, the fast decrease in the $24 \mu \mathrm{m}$ and $70 \mu \mathrm{m}$ profiles from the center to $R \simeq 2 \mathrm{kpc}$ does not occur at $160 \mu \mathrm{m}$. This is in agreement with Haas et al. (1998) who concluded from their ISO $175 \mu \mathrm{m}$ map and IRAS data that the dust near the center is relatively warm. The fast central decrease in warmer dust emission may be attributed to a decrease in the UV radiation field outside the nucleus, as a similar trend is seen in the GALEX UV profiles presented by Thilker et al. (2005). At all three IR wavelengths the arms are visible, even the weak inner arms. The bright arms forming the "10 kpc ring", are pronounced in the north and followed by an exponential decrease toward larger radii.

Although the general trend of the warm dust surface brightness (at $24 \mu \mathrm{m}$ and $70 \mu \mathrm{m}$ ) resembles more that of $\mathrm{H} \alpha$ than those of the neutral gas profiles (Fig. 6, lower panels), small variations 

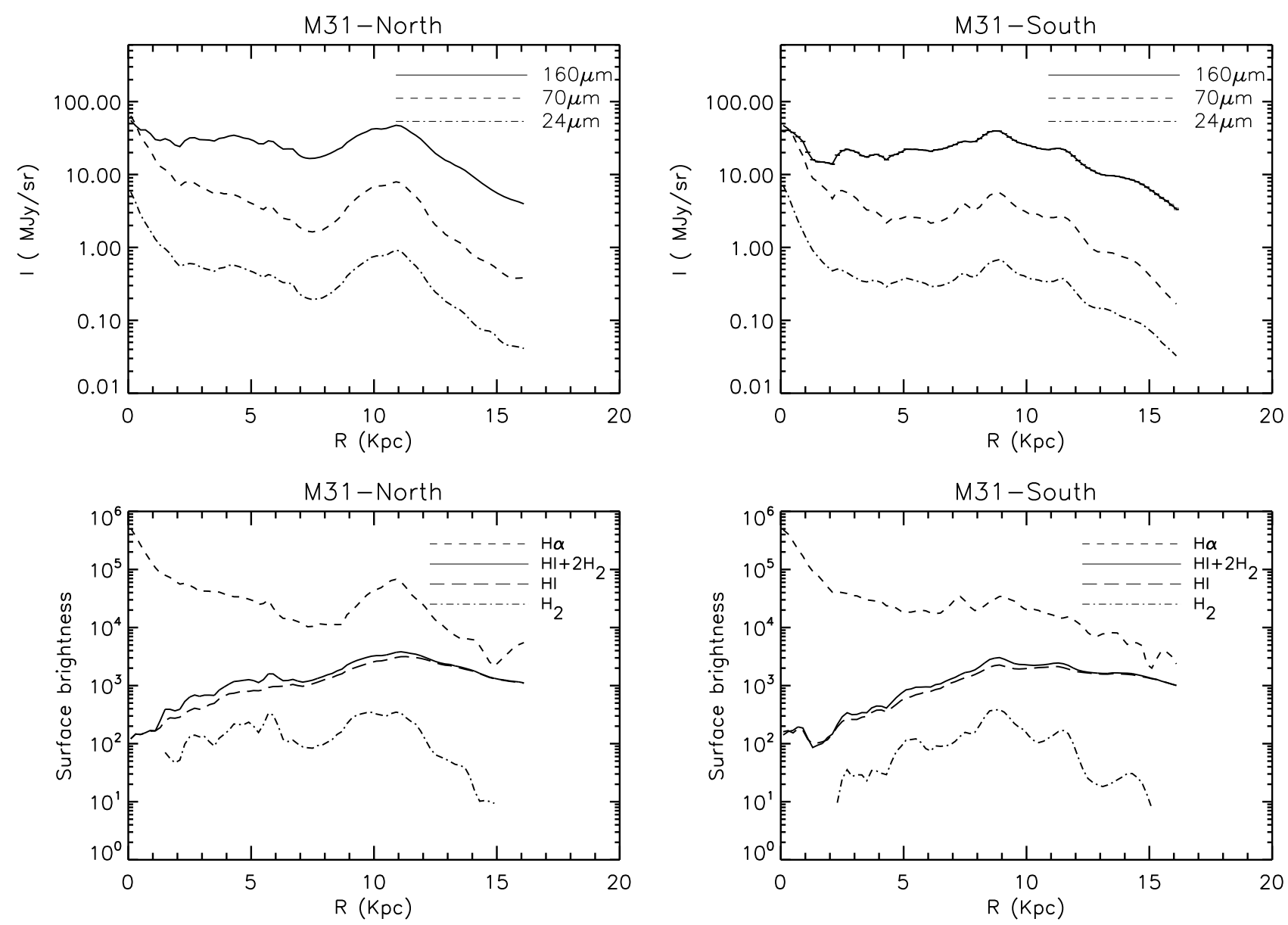

Fig. 6. Top: radial profiles of the Spitzer IR emission from the northern (left) and the southern (right) halves of M 31. Bottom: radial profiles of the surface densities of the atomic, molecular and total neutral gas together with that of the ionized gas (de-reddened $\mathrm{H} \alpha$ ) for the northern (left) and southern (right) halves of $\mathrm{M} 31$. The units are $10^{18}$ atoms cm${ }^{-2}$ for $\mathrm{HI}$ and $\mathrm{HI}+2 \mathrm{H}_{2}, 10^{18}$ molecules cm ${ }^{-2}$ for $\mathrm{H}_{2}$, and $10^{10} \mathrm{erg} \mathrm{s}^{-1} \mathrm{~cm}^{-2} \mathrm{sr}^{-1}$ for $\mathrm{H} \alpha$ profiles. The profiles show intensities along the line of sight averaged in circular rings of $0.2 \mathrm{kpc}$ width in the plane of $\mathrm{M} 31$ against the galactocentric radius. The errors are smaller than $5 \%$ for all profiles, only for $\mathrm{H}_{2}$ they increase from $10 \%$ to $25 \%$ at $R>12.3 \mathrm{kpc}$ and in the inner arms for $R<4.5 \mathrm{kpc}$.

(e.g. in the inner $5 \mathrm{kpc}$ and for $R=11-12 \mathrm{kpc}$ in the south) follow those in the total gas distribution due to variations in the molecular gas. Beyond about $5 \mathrm{kpc}$, the radial profile of the cold dust $(160 \mu \mathrm{m})$ is similar to that of the molecular gas, but with smoother variations. The minimum between $5 \mathrm{kpc}$ and $10 \mathrm{kpc}$ radius at $24 \mu \mathrm{m}$ and $70 \mu \mathrm{m}$ is less deep at $160 \mu \mathrm{m}$ and is missing in the HI profile.

We obtained radial scale lengths between the maximum in the "10 kpc ring" and $R=14.9 \mathrm{kpc}$ for the northern $\left(l_{\mathrm{N}}\right)$ and southern $\left(l_{\mathrm{S}}\right)$ halves of M 31 separately as well as for the total area $(l)$. We fit an exponential function of the form

$I(R)=I_{0} \exp (-R / l)$,

where $I_{0}$ is the intensity at $R=10.9 \mathrm{kpc}$ for the total area and in the north, and $R=8.9 \mathrm{kpc}$ in the south. The resulting scale lengths are listed in Table 3.

In each half of M 31, the scale lengths of the warm dust emission are smaller than that of the cold dust. This confirms that the warm dust is mainly heated by the UV photons from the starforming regions in the " $10 \mathrm{kpc}$ ring" and the cold dust mainly by the interstellar radiation field (ISRF) from old stars (Xu \& Helou 1996).

The scale lengths of the $24 \mu \mathrm{m}$ and $70 \mu \mathrm{m}$ emission are nearly the same and the $24 \mu \mathrm{m}$-to- $70 \mu \mathrm{m}$ intensity ratio (Fig. 7)
Table 3. Exponential scale lengths of dust and gas emissions from M 31.

\begin{tabular}{llll}
\hline \hline & $l_{\mathrm{N}}(\mathrm{kpc})$ & $l_{\mathrm{S}}(\mathrm{kpc})$ & $l(\mathrm{kpc})$ \\
\hline IR $160 \mu \mathrm{m}$ & $1.86 \pm 0.06$ & $3.87 \pm 0.18$ & $2.29 \pm 0.11$ \\
IR $70 \mu \mathrm{m}$ & $1.38 \pm 0.08$ & $3.07 \pm 0.17$ & $1.66 \pm 0.09$ \\
$\mathrm{IR} 24 \mu \mathrm{m}$ & $1.38 \pm 0.07$ & $3.44 \pm 0.21$ & $1.57 \pm 0.06$ \\
$\mathrm{H}_{2}$ & $1.14 \pm 0.07$ & $2.40 \pm 0.22$ & $1.27 \pm 0.11$ \\
$\mathrm{HI}$ & $4.54 \pm 0.24$ & $15.90 \pm 1.70$ & $5.90 \pm 0.20$ \\
$\mathrm{HI}+2 \mathrm{H}_{2}$ & $3.80 \pm 0.12$ & $9.00 \pm 0.69$ & $4.73 \pm 0.21$ \\
$\mathrm{H} \alpha$ & $1.08 \pm 0.03$ & $2.92 \pm 0.10$ & $1.39 \pm 0.04$ \\
\hline$\tau_{\mathrm{H} \alpha}$ & $2.48 \pm 0.07$ & $5.06 \pm 0.22$ & $3.17 \pm 0.15$ \\
\hline
\end{tabular}

Notes. The scale lengths were calculated from $R=10.9 \mathrm{kpc}$ to $R=14.9 \mathrm{kpc}$ for the whole galaxy $(l)$ and the northern half $\left(l_{\mathrm{N}}\right)$ and from $R=8.9 \mathrm{kpc}$ to $R=14.9 \mathrm{kpc}$ for the southern half $\left(l_{\mathrm{S}}\right)$. The scale length of $\tau_{\mathrm{H} \alpha}$ is also shown for comparison.

hardly varies between $R \simeq 10 \mathrm{kpc}$ and $R \simeq 15 \mathrm{kpc}$. This indicates a similar distribution of their origins. Assuming that the main source of the $24 \mu \mathrm{m}$ emission is very small dust grains and of the $70 \mu \mathrm{m}$ and $160 \mu \mathrm{m}$ emission is big grains, as argued by Walterbos \& Schwering (1987), a constant intensity ratio of the $24 \mu \mathrm{m}$-to-70 $\mu \mathrm{m}$ and $24 \mu \mathrm{m}$-to- $160 \mu \mathrm{m}$ emission suggests that the very small and big grains are well mixed in the interstellar 


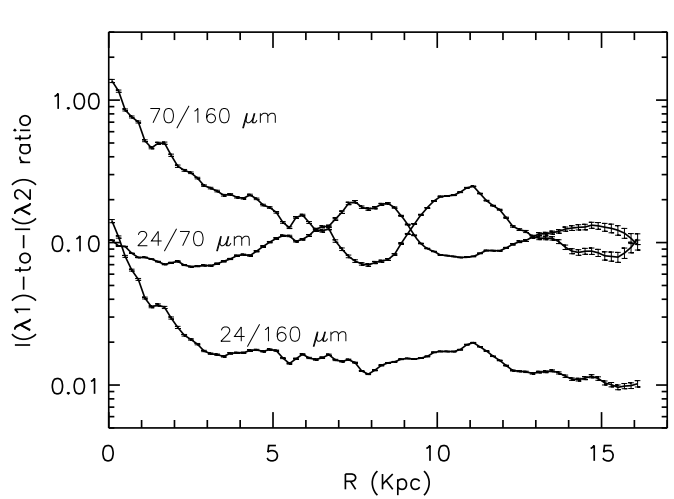

Fig. 7. Ratio of the MIPS IR intensities against galactocentric radius in M 31 .

medium. Other possible origins of the $24 \mu \mathrm{m}$ emission are stars with dust shells like evolved AGB stars or Carbon stars. Using the IRAS data, (Soifer et al. 1986) attributed the $25 \mu \mathrm{m}$ emission from the bulge (central $8^{\prime}$ ) of M 31 to circumstellar dust emission from late-type stars. In the disk of M 31, Walterbos \& Schwering (1987) found no direct evidence of a contribution from stars with dust shells (contrary to that in the Milky Way, Cox et al. 1986).

The higher resolution and sensitivity of the MIPS IR intensity ratios (Fig. 7), however, provide more information. Although at $R>3 \mathrm{kpc}$ the variations in the IR intensity ratios are not large, their radial behavior is not the same. For instance, the 24-to-70 $\mu \mathrm{m}$ intensity ratio peaks between $5 \mathrm{kpc}$ and $10 \mathrm{kpc}$ radius, whereas the 70 -to- $160 \mu \mathrm{m}$ intensity ratio peaks in the "10 kpc ring". The latter can be explained by the higher temperature of the dust heated by OB associations in the " $10 \mathrm{kpc}$ ring". The fact that the 24-to-70 $\mu \mathrm{m}$ intensity ratio is not enhanced in the "10 kpc ring" (and in the central region) shows the invalidity of this ratio for temperature determination due to the important contribution from the very small grains. On the other hand, the enhancement of the 24 -to- $70 \mu \mathrm{m}$ in regions where there is no strong radiation field (between the arms) reveals possibly different origins of the $24 \mu \mathrm{m}$ and $70 \mu \mathrm{m}$ emission. The stellar origin, e.g. photosphere of cool stars or dust shell of the evolved stars, may provide the enhancement of the 24 -to- $70 \mu \mathrm{m}$ intensity ratio in the inter-arm region. In M 33, Verley et al. (2009) attributed a similar enhancement of the diffuse $24 \mu \mathrm{m}$ emission to dusty circumstellar shells of unresolved, evolved AGB stars. For M 31, this needs to be quantified through a more detailed study and modeling of the spectral energy distribution, which is beyond the scope of this paper.

\subsection{Gas-to-dust ratio}

The gas-to-dust mass ratio and its variation across the galaxy can provide information about the metallicity distribution (e.g. Viallefond et al. 1982) and hence about the evolutionary history of the galaxy. The relative amount of dust and gas is expected to be correlated with the abundance of the heavy elements (Draine et al. 2007).

A number of authors has studied the gas-to-dust ratio in M 31 by comparing HI column densities and optical or UV extinction (Walterbos \& Kennicutt 1988; Bajaja \& Gergely 1977; Xu \& Helou 1996; Nedialkov et al. 2000; Savcheva \& Tassev 2002). All authors found an increase in the atomic gas-to-dust ratio with radius. Walterbos \& Schwering (1987) derived the
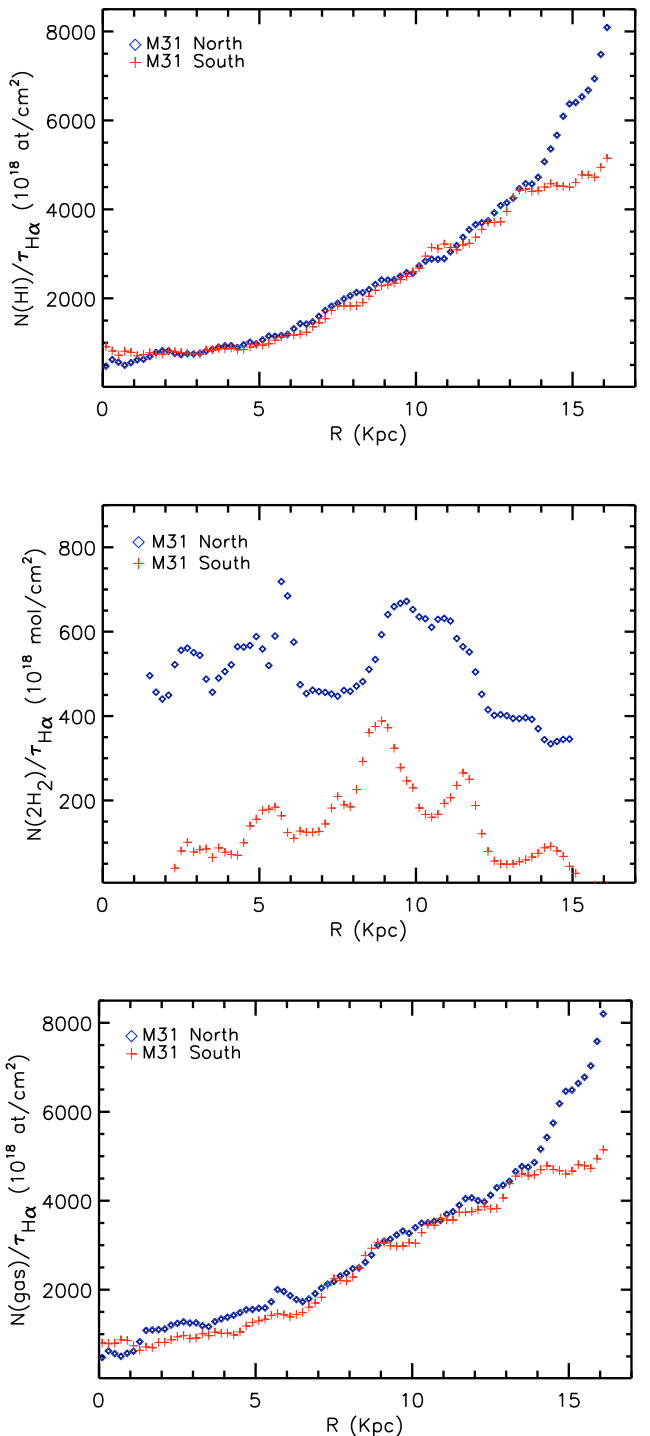

Fig. 8. Radial profiles of the gas-to-dust ratios in $\mathrm{M} 31$, the northern half and the southern half. Top: $N(\mathrm{HI}) / \tau_{\mathrm{H} \alpha}$, middle: $N\left(2 \mathrm{H}_{2}\right) / \tau_{\mathrm{H} \alpha}$, bottom: $N\left(\mathrm{HI}+2 \mathrm{H}_{2}\right) / \tau_{\mathrm{H} \alpha}$. In the middle panel, the northern profile is shifted by 300 units for clarity. The errors are smaller than $5 \%$ everywhere only for $N\left(2 \mathrm{H}_{2}\right) / \tau_{\mathrm{H} \alpha}$ they increase from $10 \%$ to $25 \%$ at $R>12.3 \mathrm{kpc}$ and in the inner arms for $R<4.5 \mathrm{kpc}$.

HI gas-to-dust ratio using dust optical depth from IRAS $60 \mu \mathrm{m}$ and $100 \mu \mathrm{m}$ data. They found a radial gradient that is 4-5 times larger than the abundance gradient of Blair et al. (1982). After adding the molecular and atomic gas column densities, Nieten et al. (2006) obtained a strong radial increase in the total gas-to$175 \mu \mathrm{m}$ intensity resulting from the increase in the atomic gasto- $175 \mu \mathrm{m}$ intensity. As the dust optical depth is a better measure for the dust column density than the temperature-dependent dust emission, we re-investigated the gas-to-dust ratio in M 31 taking advantage of the high resolution of the Spitzer MIPS data.

We calculated the radial profiles of the three gas-to-dust ratios from the mean column densities of $N(\mathrm{HI}), N\left(2 \mathrm{H}_{2}\right)$, $N\left(\mathrm{HI}+2 \mathrm{H}_{2}\right)$ and $\tau_{\mathrm{H} \alpha}$ in circular rings of $0.2 \mathrm{kpc}$ width in the plane of the galaxy. Figure 8 (upper panel) shows that the atomic gas-to-dust ratio increases exponentially with radius by more than a factor of 10 from about $0.6 \times 10^{21}$ at cm $\mathrm{cm}^{-2}$ at the center to about $6.5 \times 10^{21}$ at cm $\mathrm{cm}^{-2}$ at $R=15 \mathrm{kpc}$. The increase is surprisingly smooth and, at least up to $R=13 \mathrm{kpc}$, nearly the 
Table 4. Exponential scale lengths $L$ and radial gradients of dust-to-gas ratios and the abundance $[\mathrm{O} / \mathrm{H}]$.

\begin{tabular}{llcll}
\hline \hline Ratio & $\begin{array}{l}R \\
(\mathrm{kpc})\end{array}$ & $\mathrm{C}$ & $\begin{array}{l}L \\
(\mathrm{kpc})\end{array}$ & $\begin{array}{l}\text { Gradient } \\
(\mathrm{dex} / \mathrm{kpc})\end{array}$ \\
\hline$\tau_{\mathrm{H} \alpha} / N(\mathrm{HI})$ & $0-16$ & $1.95 \pm 0.08$ & $6.2 \pm 0.1$ & $0.070 \pm 0.001$ \\
& $5-15$ & $1.97 \pm 0.08$ & $6.1 \pm 0.2$ & $0.071 \pm 0.001$ \\
& & & & \\
$\tau_{\mathrm{H} \alpha} / N$ (gas) & $0-16$ & $1.35 \pm 0.05$ & $7.3 \pm 0.2$ & $0.059 \pm 0.002$ \\
& $5-15$ & $1.27 \pm 0.06$ & $7.4 \pm 0.2$ & $0.059 \pm 0.002$ \\
{$[\mathrm{O} / \mathrm{H}] \times 10^{-4}$} & $5-15$ & $2.95 \pm 0.35$ & $9.7 \pm 2.6$ & $0.045 \pm 0.012$ \\
\hline
\end{tabular}

Notes. Here Ratio $(R)=\mathrm{C} \cdot \exp (-R / L)$. Units of $N(\mathrm{HI})$ and $N($ gas $)$ are $10^{21} \mathrm{at} \mathrm{cm}^{-2}$. Errors are standard deviations.

same for the northern and southern half, indicating little variation between arm and inter-arm regions and within the arms. In contrast, the molecular gas-to-dust ratio (Fig. 8, middle panel) does not increase systematically with radius but shows clear enhancements of a factor $2-3$ in the spiral arms and the " $10 \mathrm{kpc}$ ring". The minima in the inter-arm regions are due to a stronger decrease in $N\left(2 \mathrm{H}_{2}\right)$ than in $\tau_{\mathrm{H} \alpha}$. Figure 3 shows that along the arms $N\left(2 \mathrm{H}_{2}\right) / \tau_{\mathrm{H} \alpha}$ also varies significantly because maxima in $\mathrm{H}_{2}$ emission and $\tau_{\mathrm{H} \alpha}$ are often not coincident. The variations in $N\left(2 \mathrm{H}_{2}\right) / \tau_{\mathrm{H} \alpha}$ are visible in the profile of the total gas-to-dust ratio (Fig. 8, bottom panel) as weak enhancements at the positions of the arms near $R=6 \mathrm{kpc}$ and $R=8-12 \mathrm{kpc}$. As the atomic gas is the dominant gas phase in M 31, dust mixed with HI gas largely determines the optical depth. Inspection of the distribution of the total gas-to-dust ratio across M 31 (not shown) reveals small-scale variations along the arms of typically a factor of 2 .

We conclude that the radial increase in the total gas-to-dust ratio of more than a factor 10 between the center and $R=15 \mathrm{kpc}$ is entirely due to that of the atomic gas-to-dust ratio, whereas the molecular gas-to-dust ratio is only increased in the arms. This confirms the conclusion of Nieten et al. (2006) based on the same gas data and the $175 \mu \mathrm{m}$ intensity.

At which radius in M 31 would the gas-to-dust ratio observed in the solar neighborhood occur? Bohlin et al. (1978) and Diplas \& Savage (1994) derived $N(\mathrm{HI}) / E(B-V)=4.8 \times$ $10^{21}$ at cm$^{-2} \mathrm{mag}^{-1}$ and $(4.9 \pm 0.3) \times 10^{21}$ at cm$^{-2} \mathrm{mag}^{-1}$, respectively, using the extinction towards large samples of stars to determine the color excess $E(B-V)$. Since $E(B-V)=$ $A_{\mathrm{V}} / R_{\mathrm{V}}$, where the visual extinction $A_{\mathrm{V}}=1.234 \tau_{\mathrm{H} \alpha}$ mag (e.g. Krügel 2003) and the total/selective extinction $R_{\mathrm{V}}=2.8 \pm 0.3$ in M 31 (Walterbos \& Kennicutt 1988), we have $E(B-$ $V)=0.44 \tau_{\mathrm{H} \alpha} \mathrm{mag}^{-1}$. Hence, a value of $N(\mathrm{HI}) / E(B-V)=$ $4.9 \times 10^{21}$ at cm $^{-2} \mathrm{mag}^{-1}$ corresponds to $N(\mathrm{HI}) / \tau_{\mathrm{H} \alpha}=2.2 \times$ $10^{21} \mathrm{at} \mathrm{cm}^{-2}$, which occurs in M 31 near $R=8.5 \mathrm{kpc}$ (Fig. 8, top panel), just in the bright emission ring. The total gas-to-dust ratio near the sun of $5.8 \times 10^{21}$ at cm$^{-2} \mathrm{mag}^{-1}$ (Bohlin et al. 1978) corresponding to $N($ gas $) / \tau_{\mathrm{H} \alpha}=2.6 \times 10^{21}$ at cm $^{-2}$ occurs at nearly the same radius (Fig. 8, bottom panel). Thus the gas-to-dust ratio near the sun is similar to that in the " $10 \mathrm{kpc}$ ring" in M 31, in agreement with earlier studies (van Genderen 1973; Walterbos \& Schwering 1987).

In contrast to Fig. 8, we present in Fig. 9 the radial profiles of the dust-to-gas ratios, here for the total area in M 31. The two lower curves closely follow exponentials with scale lengths of $6.1 \pm 0.2 \mathrm{kpc}$ and $7.4 \pm 0.2 \mathrm{kpc}$ for $\tau_{\mathrm{H} \alpha} / N(\mathrm{HI})$ and $\tau_{\mathrm{H} \alpha} / N$ (gas), respectively, between $R=5 \mathrm{kpc}$ and $R=15 \mathrm{kpc}$ (see Table 4). For nearly the same radial range $(R=3-15 \mathrm{kpc})$, Walterbos \& Schwering (1987) derived a scale length of $\tau_{100 \mu \mathrm{m}} / N(\mathrm{HI}) \simeq 4 \mathrm{kpc}$ from data near the major axis. Walterbos $\&$ Kennicutt (1988) obtained a scale length of $A_{\mathrm{B}} / N(\mathrm{HI}) \simeq 9 \mathrm{kpc}$ for the inner and outer dust lanes, and the $\tau_{\mathrm{V}} / N(\mathrm{HI})$ ratio of $\mathrm{Xu}$ $\&$ Helou (1996) for diffuse spiral arm regions also indicates a scale length of about $4 \mathrm{kpc}$ (all scale lengths were scaled to $D=780 \mathrm{kpc}$ ). Since our scale lengths are not restricted to specific areas, our results are more representative for the mean dustto-gas ratios in the disk of M 31.

As dust consists of heavy elements and both dust and heavy elements are found in star formation regions, the radial variations in the dust-to-gas ratio and the metal abundance are expected to be similar (e.g. (Hirashita 1999; Hirashita et al. 2002). This has indeed been observed in several nearby galaxies (Issa et al. 1990). In M 31 the variation in the metallicity with radius is not well established. Measurements of the element abundance strongly depend on the empirical method and calibration applied (Trundle et al. 2002). Pagel et al. (1979) showed that the $([\mathrm{OII}]+[\mathrm{OIII}]) / \mathrm{H} \beta$ ratio (the so called " $\mathrm{R}_{23}$ ") is a good probe of oxygen abundance and radial trends of this ratio have been studied in many nearby galaxies (e.g. Pagel \& Edmunds 1981; Evans 1986; Henry \& Howard 1995; Garnett et al. 1997). Blair et al. (1982) and Dennefeld \& Kunth (1981) derived $R_{23}$ for HII regions in $\mathrm{M} 31$. We combined their results and derived a scale length of $\log [\mathrm{O} / \mathrm{H}]$ of $9.7 \pm 2.6 \mathrm{kpc}$ corresponding to a gradient of $0.045 \pm 0.012 \mathrm{dex} / \mathrm{kpc}$ (Table 4). Comparing four different calibrations, Trundle et al. (2002) derived gradients of $0.027-0.013 \mathrm{dex} / \mathrm{kpc}$ using the $11 \mathrm{HII}$ regions of Blair et al. (1982), with Pagel's calibration giving $0.017 \pm 0.001 \mathrm{dex} / \mathrm{kpc}$. Since our value of the $[\mathrm{O} / \mathrm{H}]$ gradient is based on $19 \mathrm{HII}$ regions, we expect it to be more reliable than that of Trundle et al. (2002).

Table 4 shows that the radial gradient in $\tau_{\mathrm{H} \alpha} / N$ (gas) best matches the metallicity gradient. In view of the large uncertainties, the gradients in the dust-to-gas surface-density ratio and the oxygen abundance in M 31 may indeed be comparable. A much larger sample of abundance measurements of HII regions is needed to verify this similarity. Our result agrees with the approximately linear trend between gradients in dust-to-gas ratios and $[\mathrm{O} / \mathrm{H}]$ in nearby galaxies noted by Issa et al. (1990).

\section{Wavelet analysis of dust and gas emission}

To investigate the physical properties of different phases of the interstellar medium as a function of the size of emitting regions, wavelet transformation is an ideal tool. We use the Pet Hat wavelet (see Frick et al. 2001; Tabatabaei et al. 2007a) to decompose the emissions of IR, HI, H2, HI + 2H2, and dereddened $\mathrm{H} \alpha$ into 10 spatial scales starting at $0.4 \mathrm{kpc}$ (about twice the resolution). The central $2 \mathrm{kpc}$ was subtracted from all images before the wavelet transformation to prevent a strong influence of the nucleus on the results. As an example, we show the extinction-corrected $\mathrm{H} \alpha$ map and the $\mathrm{H} \alpha$ emission for 3 different scales in Fig. 10. On the scale of $0.4 \mathrm{kpc}$, the distribution of HII complexes and large HII regions is borne out. The scale of $1.6 \mathrm{kpc}$ (the typical width of spiral arms) shows connected HII complexes along the arms, and on the scale of $4 \mathrm{kpc}$ we see the extended emission from the "10 kpc ring".

\subsection{Wavelet spectra}

The wavelet spectrum, $M(a)$, represents the distribution of the emitting power as function of the scale $a$. The wavelet spectrum will smoothly increase towards larger scales if most of the emission is coming from diffuse structures forming the largest 


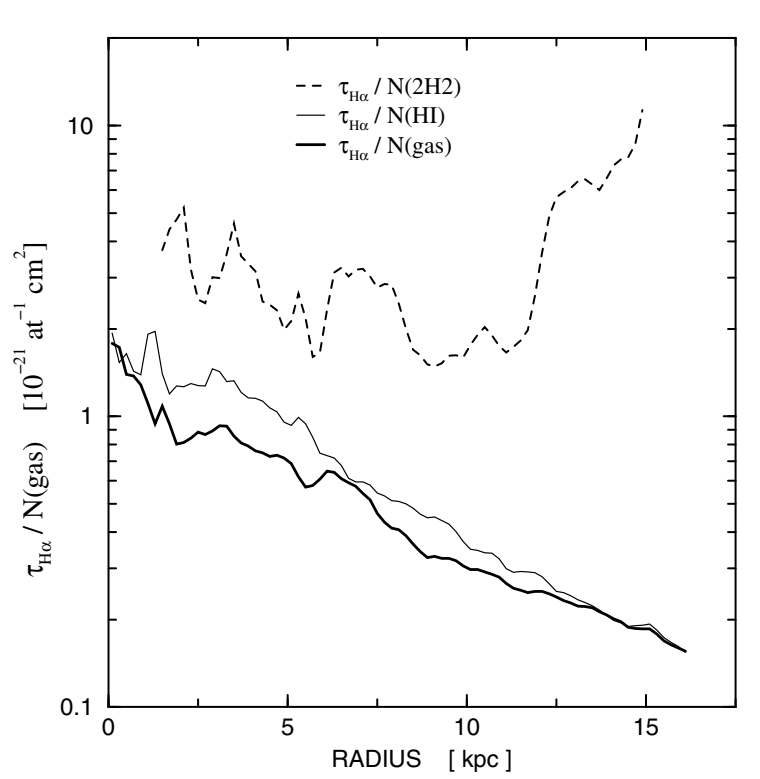

Fig. 9. Dust-to-gas ratios as function of galactocentric radius for M 31, calculated from the radial profiles of $\tau_{\mathrm{H} \alpha}, N(\mathrm{HI}), N\left(2 \mathrm{H}_{2}\right)$ and $N\left(\mathrm{HI}+2 \mathrm{H}_{2}\right)=N($ gas $)$.

scales, here up to $25 \mathrm{kpc}$. On the other hand, the spectrum will decrease with increasing scale if compact structures are the dominant source of emission. The spectra of the IR and gas emission are shown in Fig. 11.

All IR and gas spectra are intermediate between the two cases described above. Only the spectra of the HI gas and the $160 \mu \mathrm{m}$ emission generally increase with scale indicating the importance of diffuse $\mathrm{HI}$ and cold dust emission. In addition, the HI spectrum exhibits a dominant scale at $a \simeq 4 \mathrm{kpc}$ corresponding to the width of the "10 kpc ring", where strong diffuse emission occurs in interarm regions. The large width of the HI "ring" is also visible in the radial profiles in Fig. 6. The dominant scale of the emission from warm dust, molecular gas and $\mathrm{H} \alpha$ is near $1 \mathrm{kpc}$, where complexes of giant molecular clouds and starforming regions show up. The IR spectra at $24 \mu \mathrm{m}$ and $70 \mu \mathrm{m}$ on scales $a<6 \mathrm{kpc}$ look most similar indicating that the starforming regions are the main heating sources at both wavelengths. On the other hand, the effect of the ISRF heating the cold dust is well indicated in the $160 \mu \mathrm{m}$ spectrum where a general increase towards larger scales is found. All spectra, apart from that of $\mathrm{HI}$, show a minimum near $a=6 \mathrm{kpc}$ corresponding to the large, weak interarm region inside the " $10 \mathrm{kpc}$ ring". The spectrum of $\mathrm{H} \alpha$ is most similar to that of $70 \mu \mathrm{m}$, which may explain why the $\mathrm{H} \alpha$ emission correlates better with $70 \mu \mathrm{m}$ emission than with that at $24 \mu \mathrm{m}$ (see Sect. 6.2 and Table 6).

The spectrum of the $\mathrm{H} \alpha$ emission is flat on small scales up to $1.6 \mathrm{kpc}$, the width of the spiral arms in the $\mathrm{H} \alpha$ map. This is understandable as the emission from very compact HII regions is unresolved at our resolution and not many large HII complexes exist especially in the south (see the decomposed map in Fig. 10b of $a=0.4 \mathrm{kpc}$ ).

\subsection{Wavelet cross-correlations}

We derive the cross-correlation coefficients, $r_{w}(a)$, for different scales following Tabatabaei et al. (2007a). The correlation coefficients are plotted in terms of scale in Fig. 12. They show that IR emission correlates with the emission from different gas phases on most scales. In all cases, emission from structures on scales larger than $10 \mathrm{kpc}$ are best correlated. This corresponds to scales of the diameter of the "10 kpc ring" and the over-all structure of the galaxy. On medium scales, the weakest correlation occurs between $\mathrm{HI}$ and dust emission on $a=6 \mathrm{kpc}$. This scale includes areas of significant diffuse $\mathrm{HI}$ emission where the dust emission is weak interior to the "10 kpc ring" (compare also Fig. 11). On the smallest scale of $0.4 \mathrm{kpc}$, the cold dust emission is best correlated with that of the total neutral gas, while the warm dust emission at $70 \mu \mathrm{m}$ is best correlated with the ionized gas emission. Note that on this scale, the $24 \mu \mathrm{m}$ and $70 \mu \mathrm{m}$ (warm dust) emissions hardly correlate with HI $\left(r_{w(a)}<0.5\right)$ because only a small fraction of the HI emission occurs on this scale (see Fig. 11). Furthermore, the coefficients of the $70 \mu \mathrm{m}-\mathrm{H} \alpha$ correlation are higher than those of the $70 \mu$ m-neutral gas correlation on scales $a<6.3 \mathrm{kpc}$.

\section{Classical correlations between dust and gas}

The wavelet cross-correlations for different scales in Fig. 12 show on which scales the distributions of the various types of emission are significantly correlated. However, because the scale maps are normalized and information about absolute intensities is lost, they cannot be used to find quantitative relations between components of the ISM. Hence, to obtain numerical equations relating two distributions, we need classical correlations. Classical cross-correlations contain all scales that exist in a distribution. For example, the high-intensity points of the $\mathrm{H} \alpha-$ $70 \mu \mathrm{m}$ correlation in Fig. 14 represent high-emission peaks on small scales in the spiral arms (compare Fig. 10b), whereas lowintensity points represent weak emission around and between the arms on larger scales (compare Fig. 10d). The correlation coefficient of $79 \%$ is a mean of all scales, consistent with Fig. 12.

We made pixel-to-pixel correlations between the distributions of $\tau_{\mathrm{H}_{\alpha}}$ and $\mathrm{H}_{2}$, HI, total gas as well as between de-reddened $\mathrm{H} \alpha$ and $24 \mu \mathrm{m}, 70 \mu \mathrm{m}$, and $160 \mu \mathrm{m}$. We restricted the comparisons to radii where all data sets are complete, $R<50^{\prime}$ (or $11.4 \mathrm{kpc}$ ), and to intensities above $2 \times \mathrm{rms}$ noise. To reduce the influence of the gradient in the gas-to-dust ratio (see Sect. 4.2), we calculated correlations for two radial ranges: $0^{\prime}<R<30^{\prime}$ and $30^{\prime}<R<50^{\prime}$. We obtained sets of independent data points, i.e. a beam area overlap of $<5 \%$, by choosing pixels spaced by more than $1.67 \times$ the beamwidth. Since the correlated variables are not directly depending on each other, we fitted a power law to the bisector in each case (Isobe et al. 1990).

We also calculated the correlation coefficient, $r_{\mathrm{c}}$, to show how well two components are correlated, and the student-t test to indicate the statistical significance of the fit. For a number of independent points of $n>100$, the fit is significant at the $3 \sigma$ level if $t>3$. Errors in intercept $a_{\mathrm{c}}$ and slope $b$ of the bisector are standard deviations $(1 \sigma)$.

We first discuss the correlations between the neutral gas and dust extinction, scaled from $\tau_{\mathrm{H}_{\alpha}}$. Then we investigate the relationships between the emissions from dust and ionized gas. The results are given in Tables 5 and 6, and examples of correlation plots are shown in Figs. 13 to 15.

\subsection{Correlation between neutral gas and dust extinction}

In search of a general relationship between neutral gas and dust extinction, a number of authors employed scatter plots between gas column densities and extinction, optical depth or FIR surface brightness (e.g. Savage et al. 1978; Boulanger et al. 1996; 

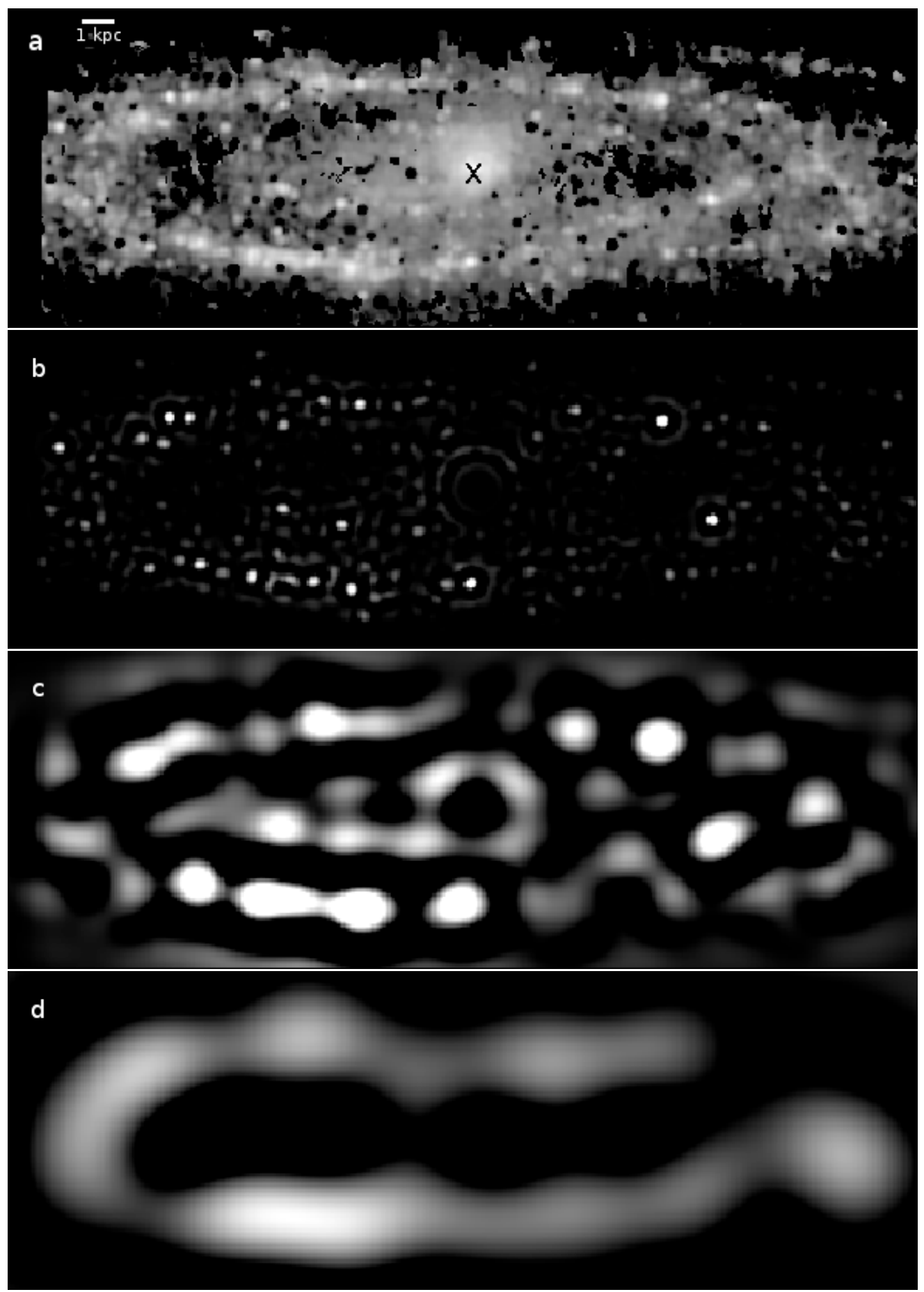

Fig. 10. Distribution of the de-reddened $\mathrm{H} \alpha$ emission a) and the wavelet decomposition for scales $0.4,1.6,4.0 \mathrm{kpc}$ b) to d). The central $2 \mathrm{kpc}$ was subtracted from the $\mathrm{H} \alpha$ map before the decomposition. The cross in the $\mathrm{H} \alpha$ map indicates the location of the center.

Walterbos \& Kennicutt 1988; Xu \& Helou 1996; Neininger et al. 1998; Nieten et al. 2006). They obtained nearly linear relationships between these quantities. As the studies on M 31 have various shortcomings (lower limits for extinction, $\mathrm{H}_{2}$ data not included and/or low angular resolution), we calculated classical correlations between the distribution of dust extinction $A_{\mathrm{H} \alpha}=$ $1.086 \tau_{\text {eff }}$ and those of $N(\mathrm{HI}), N\left(2 \mathrm{H}_{2}\right)$ and $N($ gas $)$ at our resolution of $45^{\prime \prime}$. As the correlations are restricted to gas column densities above $2 \times$ the rms noise, values of $\tau_{\mathrm{H}_{\alpha}}<0.04$ are not included (see upper panel of Fig. 13). The bisector fits given in Table 5 are plotted in the bottom panel of Fig. 13.
The relationships between $A_{\mathrm{H} \alpha}$ and $N\left(2 \mathrm{H}_{2}\right)$ for the two radial ranges are the same within errors, so the two areas can be combined. With a correlation coefficient of $r_{\mathrm{c}} \simeq 0$. 6 , the correlation is not very good, indicating that only a small part of the extinction is caused by dust in molecular clouds. This is not surprising in view of the low molecular gas fraction in M 31 (see lower panels of Fig. 6) and the small area filling factor of the molecular gas compared to that of the atomic gas.

The correlations between $A_{\mathrm{H} \alpha}$ and $N(\mathrm{HI})$ are indeed better $\left(r_{\mathrm{c}} \simeq 0.7\right)$ than those between $A_{\mathrm{H} \alpha}$ and $N\left(2 \mathrm{H}_{2}\right)$, but the relationships for the two radial intervals are not the same. Although 

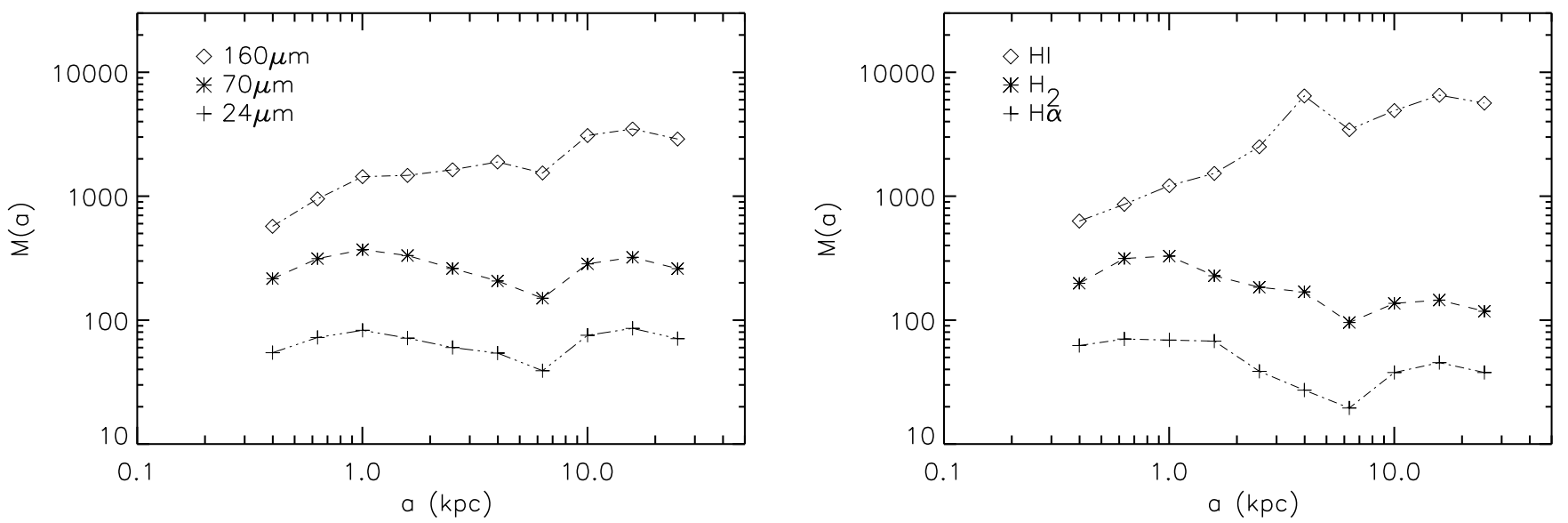

Fig. 11. Wavelet spectra of MIPS IR (left) and gas (right) emission in M 31, shown in arbitrary units. The data points correspond to the scales 0.4 , $0.6,1.0,1.6,2.5,4.0,6.3,10.0,15.9,25.1 \mathrm{kpc}$.

Table 5. Power-law relations and correlation coefficients $r_{\mathrm{c}}$ between dust extinction and gas components.

\begin{tabular}{lllccccc}
\hline \hline $\begin{array}{l}X \\
\left(10^{18} \text { at cm }\right.\end{array}$ & $Y$ & $R$ & $\left.a_{\mathrm{c}}\right)$ & $b$ & $n$ & $r_{\mathrm{c}}$ & $t$ \\
\hline$N\left(2 \mathrm{H}_{2}\right)$ & $A_{\mathrm{H} \alpha}$ & $0-30$ & $-1.88 \pm 0.06$ & $0.53 \pm 0.03$ & 207 & $0.60 \pm 0.06$ & 11 \\
& & $30-50$ & $-1.83 \pm 0.04$ & $0.52 \pm 0.02$ & 610 & $0.64 \pm 0.03$ & 20 \\
& & $0-50$ & $-1.85 \pm 0.03$ & $0.52 \pm 0.01$ & 817 & $0.63 \pm 0.03$ & 23 \\
$N(\mathrm{HI})$ & $\mathrm{A}_{\mathrm{H} \alpha}$ & $0-30$ & $-3.29 \pm 0.11$ & $0.94 \pm 0.04$ & 354 & $0.69 \pm 0.04$ & 18 \\
& & $30-50$ & $-3.26 \pm 0.07$ & $0.84 \pm 0.02$ & 768 & $0.72 \pm 0.03$ & 29 \\
& & & & & & & \\
$N($ gas $)$ & $\mathrm{A}_{\mathrm{H} \alpha}$ & $0-30$ & $-2.97 \pm 0.08$ & $0.79 \pm 0.03$ & 350 & $0.80 \pm 0.03$ & 25 \\
& & $30-50$ & $-3.05 \pm 0.06$ & $0.76 \pm 0.02$ & 766 & $0.77 \pm 0.02$ & 33 \\
\hline
\end{tabular}

Notes. Ordinary least-squares fits of bisector $\log (Y)=a_{\mathrm{c}}+b \log (X)$ through $n$ pairs of $(\log X, \log Y)$, where $n$ is the number of independent points (Isobe et al. 1990); $t$ is the student-t test.

both are nearly linear (power-law exponent $b \simeq 0.9$ ), their power laws are shifted (see Fig. 13) in the sense that the values of $A_{\mathrm{H} \alpha}$ in $R=30^{\prime}-50^{\prime}$ are about a factor of 2 lower than those inside $R=30^{\prime}$. This difference is caused by the radial decrease in $\tau_{\mathrm{H}_{\alpha}} / N(\mathrm{HI})$ discussed in Sect. 4.2. The variation of this ratio within each of the radial intervals contributes to the spread in the scatter plots and reduces the correlation coefficients.

The correlations between total gas $N$ (gas) and $\mathrm{A}_{\mathrm{H} \alpha}$ are best ( $r_{\mathrm{c}} \simeq 0.8$ ), as $A_{\mathrm{H} \alpha}$ represents dust mixed with both $\mathrm{HI}$ and $\mathrm{H}_{2}$. They are close to linear $(b \simeq 0.8)$ and differ by nearly a factor 2 in $A_{\mathrm{H} \alpha}$. The scatter plots for the two intervals are shown in the upper panel of Fig. 13. In linear plots both power-law fits are going through zero, suggesting that the dust causing the extinction and neutral gas are mixed down to very low densities.

Interestingly, extinction (or dust opacity) is proportional to the square root of $N\left(2 \mathrm{H}_{2}\right)$, while it is about linearly related to the atomic gas density. This is due to the quadratic dependence of $N\left(2 \mathrm{H}_{2}\right)$ on $N(\mathrm{HI})$ in $\mathrm{M} 31$ observed by (Nieten et al. 2006). This dependence is expected if in cool, dense, and dusty HI clouds the formation and destruction rates of $\mathrm{H}_{2}$ are balanced (Reach \& Boulanger 1998).

\subsection{Correlation between ionized gas and dust}

Because the emission from ionized gas is a good tracer of the present-day star formation rate and massive stars both heat the dust and ionize the gas, a correlation between the emissions from warm dust and ionized gas is expected. Relationships between the emission at $24 \mu \mathrm{m}$ and $\mathrm{Pa} \alpha$ or $\mathrm{H} \alpha$ emission from HII regions in nearby galaxies as well as relationships between global luminosities of galaxies have been reported (see Kennicutt et al. 2009, and references therein).

For M 31, the correlation between the emission from dust and ionized gas was first tested by Hoernes et al. (1998), who found a good, nearly linear correlation between warm dust emission and free-free radio emission for the radial range $30^{\prime}<R<$ $90^{\prime}$, using HIRAS data and multi-wavelength radio data. Here we correlate the extinction-corrected $\mathrm{H} \alpha$ emission presented in Fig. 10a with dust emission in the MIPS maps.

The wavelet correlations in Fig. 12 (bottom-right) show that in $\mathrm{M} 31 \mathrm{H} \alpha$ emission is best correlated with dust emission at $70 \mu \mathrm{m}$. This suggests that of the MIPS bands, the $70 \mu \mathrm{m}$ emission could best be used as the tracer of present-day star formation, making a numerical relation between the emissions at $70 \mu \mathrm{m}$ and $\mathrm{H} \alpha$ of interest. Table 6 gives the bisector fits for the two radial ranges, which are very similar. Therefore, we present this correlation in Fig. 14 for the entire radial range of $0^{\prime}<R<50^{\prime}$. The power-law fit for this radial interval is

$\log \left(I_{70}\right)=(-0.98 \pm 0.02)+(1.09 \pm 0.02) \log \left(I_{\mathrm{H} \alpha}\right)$,

where $I_{70}$ is in MJy/sr and $I_{\mathrm{H} \alpha}$ in $10^{-7} \mathrm{erg} \mathrm{s}^{-1} \mathrm{~cm}^{-2} \mathrm{sr}^{-1}$. The correlation is quite good $\left(r_{\mathrm{c}} \simeq 0.8\right)$ and nearly linear. In a linear plot 

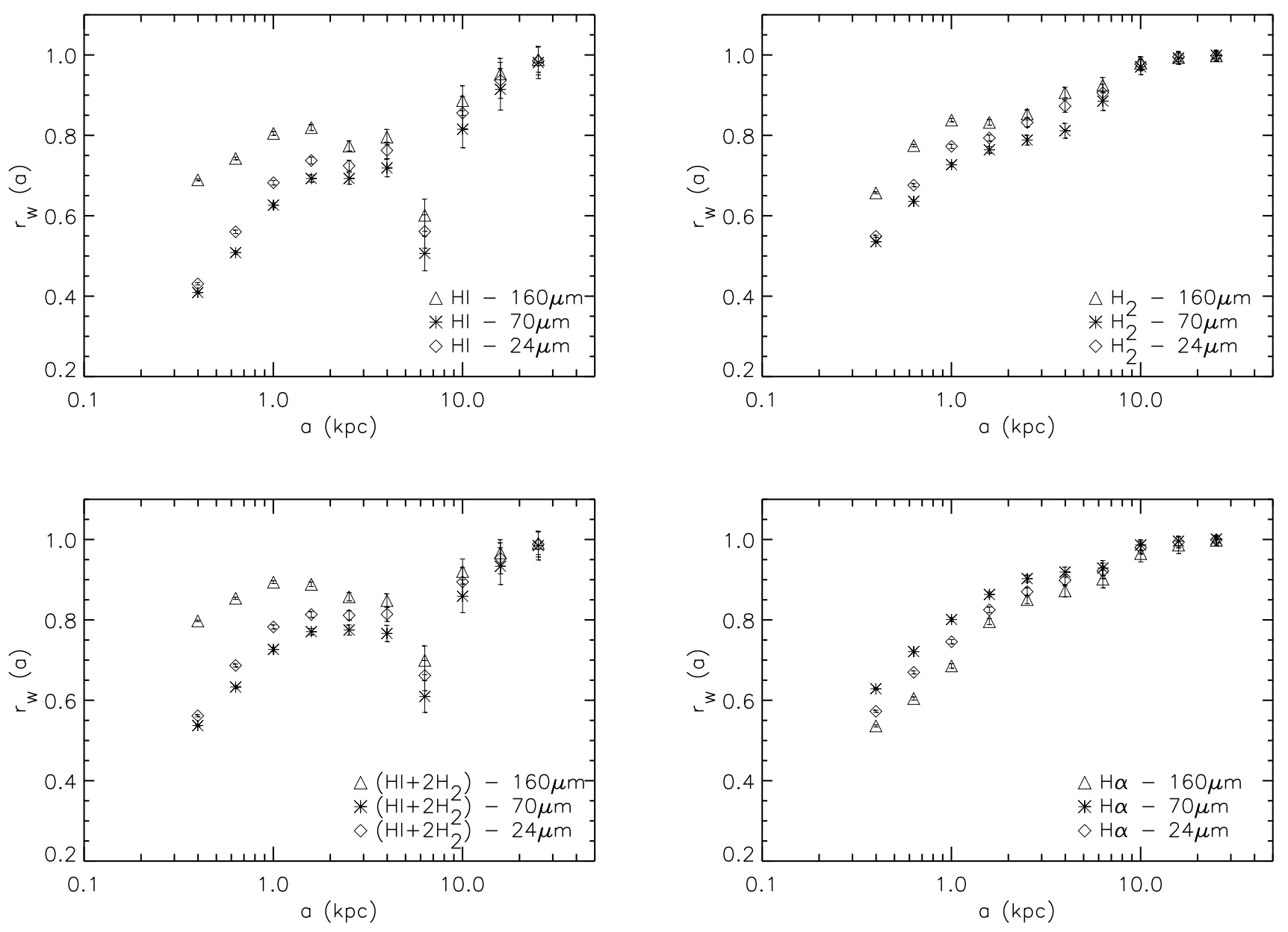

Fig. 12. Wavelet cross correlations of atomic gas (top-left), molecular gas (top-right), and total neutral gas (bottom-left) with IR emission in M 31. The IR correlation with the ionized gas (bottom-right) is also shown. The data points correspond to the scales $0.4,0.6,1.0,1.6,2.5,4.0,6.3,10.0$, 15.9 , and $25.1 \mathrm{kpc}$.

the power-law fit goes through zero suggesting that the correlation is also valid for the lowest intensities. The good correlation indicates that the heating sources that power dust emission at $70 \mu \mathrm{m}$ and ionize the gas must indeed be largely the same.

Naturally, $\mathrm{H} \alpha$ emission is less correlated with the emission from cold dust at $160 \mu \mathrm{m}$ than with emission from warm dust seen at the shorter wavelengths. This is especially so at $R<30^{\prime}$ where the radial profiles differ most (see Fig. 6). Moreover, the relation between the emission from cold dust and $\mathrm{H} \alpha$ is nonlinear (see the bisector slope $b$ in Table 6).

Table 6 shows that the correlations with $24 \mu \mathrm{m}$ are slightly worse than those with $70 \mu \mathrm{m}$. In contrast, in M 33 the $24 \mu \mathrm{m}-\mathrm{H} \alpha$ correlation is better than the $70 \mu \mathrm{m}-\mathrm{H} \alpha$ correlation (Tabatabaei et al. 2007a). This may suggest that in early-type galaxies like M 31 the contribution from evolved AGB stars to the $24 \mu \mathrm{m}$ emission is larger than in late-type galaxies like M 33. A significant stellar contribution to the $24 \mu \mathrm{m}$ emission from M 31 is also indicated by the enhancement of the $24 \mu \mathrm{m}$-to- $70 \mu \mathrm{m}$ intensity ratio in inter-arm regions where the radiation field is weak (Fig. 7).

Across M 31, the $24 \mu \mathrm{m}$ emission is linearly proportional to the extinction-corrected $\mathrm{H} \alpha$ emission $(b=0.98 \pm 0.02)$. A linear relationship was also found between the luminosities at $24 \mu \mathrm{m}$ and extinction-corrected $\mathrm{Pa} \alpha$ of HII regions in M 51 (Calzetti et al. 2005) and between the luminosities at $24 \mu \mathrm{m}$ and extinction-corrected $\mathrm{H} \alpha$ of HII regions in M 81 (Pérez-González et al. 2006). Comparing the $24 \mu \mathrm{m}$ luminosities and corrected $\mathrm{H} \alpha$ luminosities of HII regions in 6 nearby galaxies (including M 51 and M 81), Relaño et al. (2007) obtained a somewhat steeper power law with index $1.21 \pm 0.01$, in agreement with the index for global luminosities of galaxies (see also Calzetti et al. 2007). Thus, while the $L_{24}-L_{\mathrm{H} \alpha}$ relationship is linear within a single galaxy, the relationships for HII regions in a sample of galaxies and for global luminosities are non-linear. According to Kennicutt et al. (2009), the steepening is due to variations between galaxies in the contribution from evolved, non-ionizing stars to the heating of the dust that emits at $24 \mu \mathrm{m}$.

\section{Star formation rate and efficiency}

Over the last 40 years many authors have studied the relationship between the rate of star formation and gas density in M 31 by comparing the number surface density of massive young stars or of HII regions with that of HI (Berkhuijsen 1977; Tenjes \& Haud 1991; Unwin 1980; Nakai \& Sofue 1984). They found powerlaw exponents near 2 as was also obtained for the solar neighborhood by Schmidt (1959), who first proposed this relationship with HI volume density. Kennicutt (1998a) showed that a similar relationship is expected between SFR and gas column densities. The early studies suffered from the effects of dust absorption and 
F. S. Tabatabaei and E. M. Berkhuijsen: Dust, gas, and SFR in M 31

Table 6. Power-law relations and correlation coefficients $r_{\mathrm{c}}$ between the emission from dust and ionized gas.

\begin{tabular}{lllccccc}
\hline \hline $\begin{array}{l}X \\
\left(10^{-7} \mathrm{erg} \mathrm{s}^{-1} \mathrm{~cm}^{-2} \mathrm{sr}^{-1}\right)\end{array}$ & $Y$ & $R$ & $a_{\mathrm{c}}$ & $b$ & $n$ & $r_{\mathrm{c}}$ & $t$ \\
\hline$I_{\mathrm{H} \alpha}$ & $I_{24 \mu \mathrm{m}}$ & $0-30$ & $-1.76 \pm 0.05$ & $0.94 \pm 0.03$ & 417 & $0.79 \pm 0.03$ & 27 \\
& & $30-50$ & $-1.75 \pm 0.05$ & $1.00 \pm 0.03$ & 677 & $0.75 \pm 0.03$ & 30 \\
& & $0-50$ & $-1.75 \pm 0.05$ & $0.98 \pm 0.02$ & 1094 & $0.76 \pm 0.02$ & 38 \\
$I_{\mathrm{H} \alpha}$ & & & & & & \\
& $I_{70 \mu \mathrm{m}}$ & $0-30$ & $-1.04 \pm 0.03$ & $1.10 \pm 0.03$ & 412 & $0.83 \pm 0.03$ & 30 \\
& & $30-50$ & $-0.96 \pm 0.03$ & $1.09 \pm 0.03$ & 677 & $0.78 \pm 0.02$ & 32 \\
$I_{\mathrm{H} \alpha}$ & & $0-50$ & $-0.98 \pm 0.02$ & $1.09 \pm 0.02$ & 1089 & $0.79 \pm 0.02$ & 43 \\
& & & & & & & \\
& $I_{160 \mu \mathrm{m}}$ & $0-30$ & $0.26 \pm 0.05$ & $0.76 \pm 0.03$ & 417 & $0.54 \pm 0.04$ & 13 \\
& & $30-50$ & $0.47 \pm 0.04$ & $0.72 \pm 0.02$ & 678 & $0.73 \pm 0.03$ & 28 \\
\hline
\end{tabular}

Notes. Ordinary least-squares fits of bisector $\log (Y)=a_{\mathrm{c}}+b \log (X)$ through $n$ pairs of $(\log X, \log Y)$, where $n$ is the number of independent points (Isobe et al. 1990); $t$ is the student-t test.

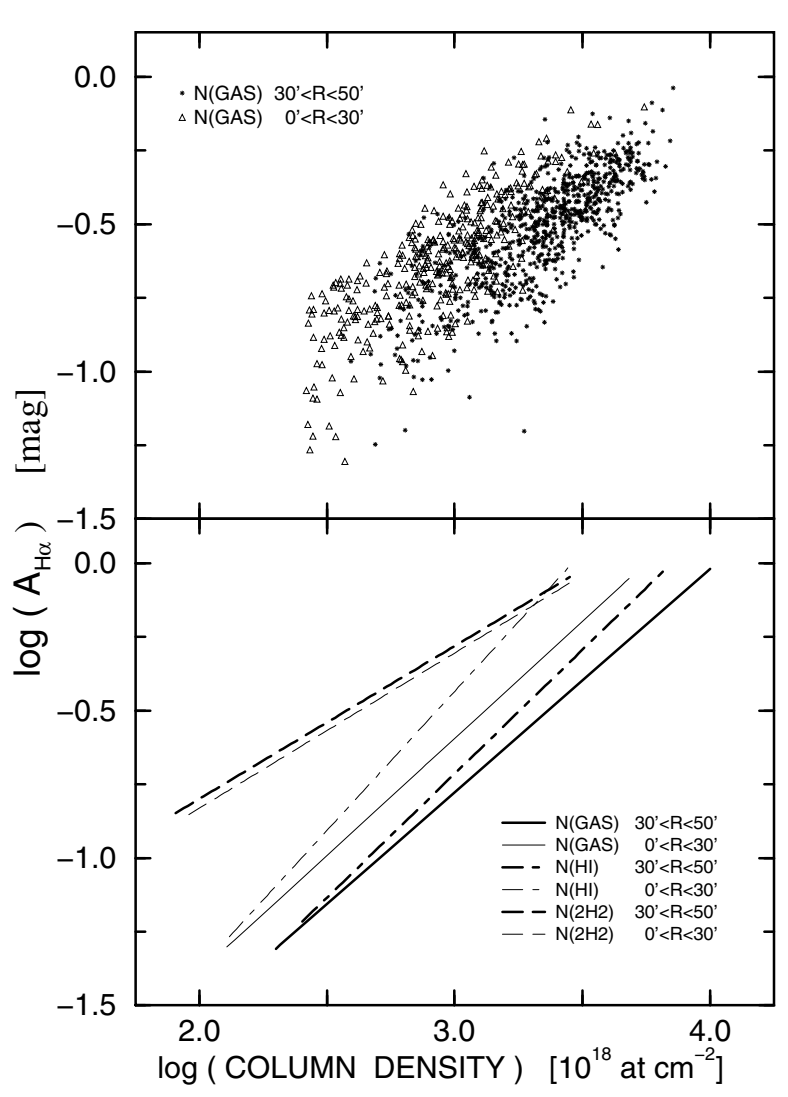

Fig. 13. Classical cross-correlations between gas column densities and dust extinction $\mathrm{A}_{\mathrm{H} \alpha}$ in the radial ranges $0^{\prime}<R<30^{\prime}$ and $30^{\prime}<R<50^{\prime}$ (or $6.8 \mathrm{kpc}<R<11.4 \mathrm{kpc}$ ). Only independent data points (separated by $1.67 \times$ beamwidth) with values above $2 \times$ rms noise were used. Top. As an example, the scatter plot between $N$ (gas) and $\mathrm{A}_{\mathrm{H} \alpha}$. Bottom. Powerlaw fits to the various correlations given in Table 5. Thick lines: $30^{\prime}<$ $R<50^{\prime}$; thin lines: $0^{\prime}<R<30^{\prime}$. The shift between these intervals of the fits to the $N(\mathrm{HI})-\mathrm{A}_{\mathrm{H} \alpha}$ and $N(\mathrm{gas})-\mathrm{A}_{\mathrm{H} \alpha}$ correlations is due to the radial increase in the atomic gas-to-dust ratio.

could not consider molecular gas (apart from Tenjes \& Haud 1991). As the necessary data are now available, we again address this issue.

We compared the distribution of the $\mathrm{H} \alpha$ emission corrected for dust attenuation (see Fig. 10a) with those of $\mathrm{HI}, \mathrm{H}_{2}$, and total gas. The corrected $\mathrm{H} \alpha$ emission is a good measure for the

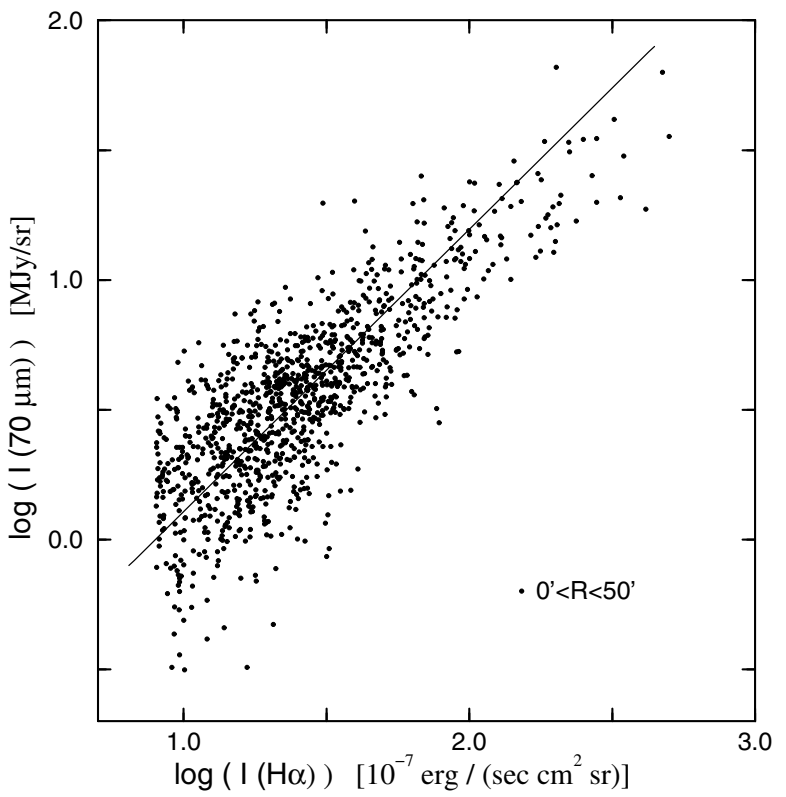

Fig. 14. Scatter plot between the surface brightnesses of ionized gas and dust emission at $70 \mu \mathrm{m}$ for the radial range $0^{\prime}-50^{\prime}$. Only independent data points (separated by $1.67 \times$ beamwidth) with values above $2 \times \mathrm{rms}$ noise are included. The line shows the power-law fit given in Table 6 , which has an exponent close to 1 . In a linear frame, this fit goes through the zero point of the plot.

present-day star formation rate (SFR) that we first estimate for the total area observed using the relation of (Kennicutt 1998b):

$\operatorname{SFR}\left(M_{\odot} \mathrm{yr}^{-1}\right)=\frac{L_{\mathrm{H}_{\alpha}}\left(\mathrm{erg} \mathrm{s}^{-1}\right)}{1.26 \times 10^{41}}$

where $L_{\mathrm{H}_{\alpha}}$ is the $\mathrm{H}_{\alpha}$ luminosity. In an area of $110.0^{\prime} \times 38.5^{\prime}$ $(R<17 \mathrm{kpc})$, the luminosity of the de-reddened $\mathrm{H}_{\alpha}$ emission is $L_{\mathrm{H}_{\alpha}}=4.75 \times 10^{40} \mathrm{erg} \mathrm{s}^{-1}$ or $L_{\mathrm{H}_{\alpha}}=1.7 \times 10^{7} L_{\odot}$ for the distance to $\mathrm{M} 31$ of $780 \mathrm{kpc}$ (see Table 1), giving $S F R=0.38 M_{\odot} \mathrm{yr}^{-1}$. However, this value is rather uncertain for two reasons. First, the contribution from the inner disk $\left(R<25^{\prime}\right.$, nearly $\left.6 \mathrm{kpc}\right)$ is overestimated because in this area the number of ionizing stars is low and the gas must be mainly heated by other sources (see Sect. 7.1). Second, our $\mathrm{H} \alpha$ map is limited to about 55' $(12.5 \mathrm{kpc})$ along the major axis, so some of the emission between $R=12.5 \mathrm{kpc}$ and $R=17 \mathrm{kpc}$ is missing. Subtracting 


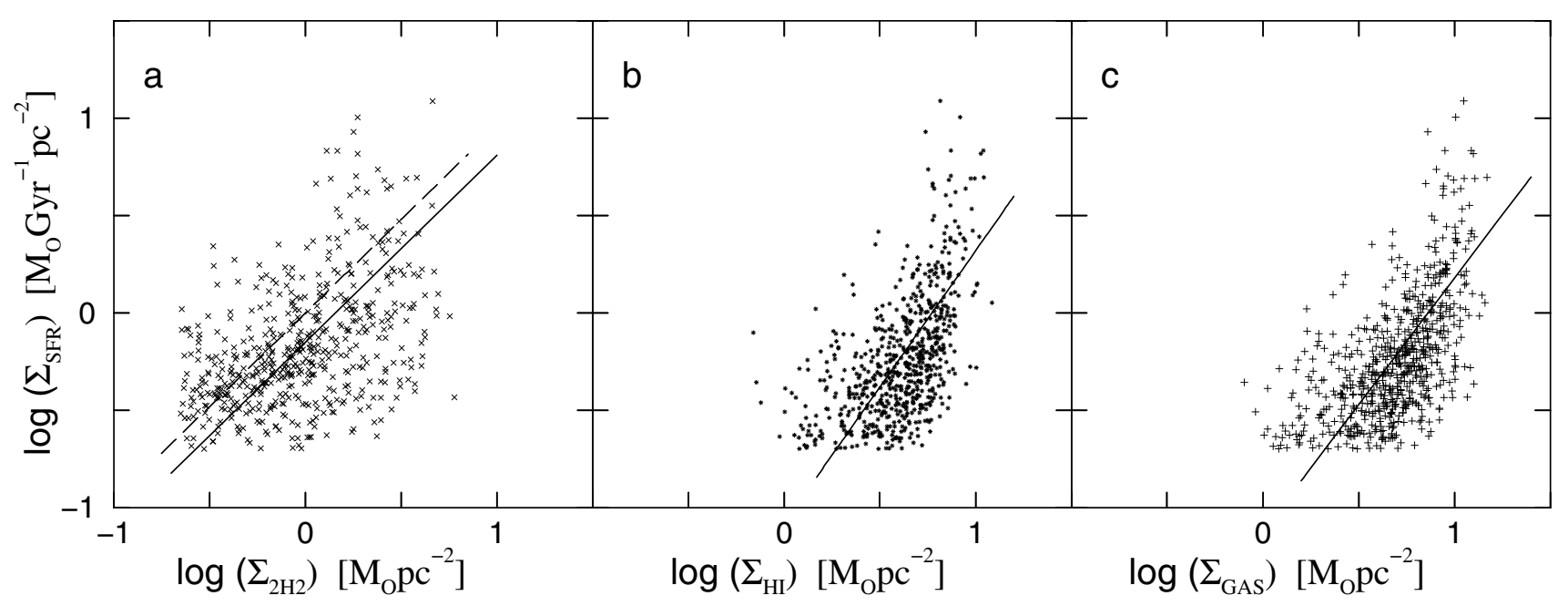

Fig. 15. Scatter plots between the surface density of the star formation rate and neutral gas surface densities for the radial interval $30^{\prime}<R<50^{\prime}$ $(6.8 \mathrm{kpc}<R<11.4 \mathrm{kpc}$ ). All surface densities are face-on values. Only independent data points (separated by $1.67 \times$ beamwidth) above $2 \times$ rms noise were used. a) SFR versus molecular gas; b) SFR versus atomic gas; c) SFR versus total gas. Full lines indicate the power-law fits given in Table 6. The relationship in a. is linear and nearly the same as the average relationship for 7 bright galaxies (dashed line) derived by Bigiel et al. (2008). Note that the cut off in $\Sigma_{\mathrm{HI}}$ and $\Sigma_{\mathrm{GAS}}$ is near $10 M_{\odot} \mathrm{pc}^{-2}$.

the luminosity from the area $R<6 \mathrm{kpc}$ gives a lower limit to the $S F R$ of $0.27 M_{\odot} \mathrm{yr}^{-1}$ for the radial range $6<R<17 \mathrm{kpc}$. Earlier estimates of the recent SFR for a larger part of the disk indicated 0.35-1 $M_{\odot} \mathrm{yr}^{-1}$ (Walterbos \& Braun 1994; Williams 2003; Barmby et al. 2006). Recently, Kang et al. (2009) derived a $S F R$ of $0.43 M_{\odot} \mathrm{yr}^{-1}$ (for metallicity $2.5 \times$ Solar) from UV observations of young starforming regions $(<10 \mathrm{Myr})$ within $120^{\prime}$ from the center $(R<27 \mathrm{kpc})$. Their Fig. 13 suggests that about $20 \%$ of this $S F R$ is coming from $R>17 \mathrm{kpc}$ and a negligible amount from $R<6 \mathrm{kpc}$. So for the range $6 \mathrm{kpc}<R<17 \mathrm{kpc}$ they find a $S F R$ of about $0.34 M_{\odot} \mathrm{yr}^{-1}$, which is consistent with our lower limit of $0.27 M_{\odot} \mathrm{yr}^{-1}$.

A $S F R$ of $0.3 M_{\odot} \mathrm{yr}^{-1}$ yields a mean face-on surface density of $\Sigma_{\mathrm{SFR}}=0.4 M_{\odot} \mathrm{Gyr}^{-1} \mathrm{pc}^{-2}$ between $R=6 \mathrm{kpc}$ and $R=17 \mathrm{kpc}$. This is about 6 times lower than the value of $\Sigma_{\mathrm{SFR}}=2.3 M_{\odot} \mathrm{Gyr}^{-1} \mathrm{pc}^{-2}$ that Verley et al. (2009) obtained for the disk of M $33(R<7 \mathrm{kpc})$, also using de-reddened $\mathrm{H} \alpha$ data.

We can also calculate the star formation efficiency between $R=6 \mathrm{kpc}$ and $R=17 \mathrm{kpc}$ in M31. The total molecular gas mass in the entire area of $R<17 \mathrm{kpc}$ in M 31 is $M\left(\mathrm{H}_{2}\right)=3.6 \times 10^{8} M_{\odot}$ (Nieten et al. 2006) and that in the area $6 \mathrm{kpc}<R<17 \mathrm{kpc}$ is $M\left(\mathrm{H}_{2}\right)=2.9 \times 10^{8} M_{\odot}$. Hence the star formation efficiency SFE $=S F R / M\left(\mathrm{H}_{2}\right)$ between $6 \mathrm{kpc}$ and $17 \mathrm{kpc}$ radius is $\mathrm{SFE}=0.9 \mathrm{Gyr}^{-1}$. It is equivalent to a molecular depletion time scale of 1.1 Gyr. Hence, the disk of M 31 is about three times less efficient in forming young massive stars than the northern part of the disk of M 33 (Gardan et al. 2007).

\subsection{Star formation rate in the " $10 \mathrm{kpc}$ ring"}

The radial distributions of $\mathrm{H} \alpha$ emission in the bottom panels of Fig. 6 show a steep decrease from the center to $R \simeq 2 \mathrm{kpc}$, followed by a shallower decrease to a minimum near $R=6 \mathrm{kpc}$. In the radial profile of $\Sigma_{\mathrm{SFR}}$, shown in the upper panel of Fig. 17 for the total area, the inner arms at $R=2.5 \mathrm{kpc}$ and $R=5.5 \mathrm{kpc}$ are only visible as little wiggles superimposed onto a high background. Clearly, the starforming regions in these arms hardly contribute to the ionization of the gas at $R<6 \mathrm{kpc}$.
Devereux et al. (1994) noted that at these radii the $\mathrm{H} \alpha$ emission is filamentary and unlike that in starforming regions, and since not many young, massive, ionizing stars are found interior to the "10 kpc ring" (Berkhuijsen \& Humphreys 1989; Tenjes \& Haud 1991; Kang et al. 2009) the gas must be ionized by other sources. Naturally, the same holds for the heating of the warm dust, the emission of which also strongly increases towards the center. In an extensive discussion, Devereux et al. (1994) concluded that a collision with another galaxy in the past may explain the ionization of the gas and the heating of the dust as well as several other peculiarities (e.g. the double nucleus) in the inner disk of M 31 (see also Block et al. 2006). We note that the UV emission may also be influenced by this event, because it shows a similarly steep increase towards the center as the $\mathrm{H} \alpha$ emission. Furthermore, all radial profiles that increase towards the center are anti- correlated with the radial profiles of $\mathrm{HI}$ and total gas (see Fig. 6, bottom panels). This leads to apparent deviations in the Kennicutt-Schmidt law in the inner disk if $\Sigma_{\mathrm{SFR}}$ is calculated from the usual star formation tracers (see Boissier et al. 2007; Yin et al. 2009).

If massive stars are not responsible for the ionization of the gas and the heating of the dust, we can neither use the $\mathrm{H} \alpha$ emission nor the infrared emission as tracers of presentday star formation at $R<6 \mathrm{kpc}$ in M 31, as was also pointed out by Devereux et al. (1994). Therefore, we investigated the relationship between SFR and neutral gas only for the interval $30^{\prime}<R<50^{\prime}(R=6.8-11.4 \mathrm{kpc})$ containing the " $10 \mathrm{kpc}$ ring".

The correlation plots in Fig. 15 and the results in Table 7 show that $\Sigma_{\text {SFR }}$ is not well correlated with the surface densities of either $\mathrm{H}_{2}$, HI or total gas $\left(r_{\mathrm{c}} \sim 0.45-0.59\right)$. In spite of this, the fitted bisectors are statistically significant $(t>3)$. Interestingly, we find a linear relationship between $\Sigma_{\mathrm{SFR}}$ and $\Sigma_{2 \mathrm{H}_{2}}$ (exponent $b=0.96 \pm 0.03$ ), which closely agrees with the average relationship for 7 nearby galaxies, much brighter than M 31 (see Fig. 15a), analyzed by Bigiel et al. (2008). While in these galaxies molecular hydrogen is the dominant gas phase, most of the neutral gas in M 31 is atomic (compare Fig. 15a,b). Hence, the surface density of SFR is linearly related to that of molecular gas, irrespective of the fraction of molecular gas or the absolute 
Table 7. Kennicutt-Schmidt law in M 31 for $30^{\prime}<R<50^{\prime}$.

\begin{tabular}{lcclcl}
\hline \hline$\Sigma\left(M_{\odot} \mathrm{pc}^{-2}\right)$ & $a_{\mathrm{c}}$ & $b$ & $n$ & $r_{\mathrm{c}}$ & $t$ \\
\hline$\Sigma_{2 \mathrm{H}_{2}}$ & $-0.15 \pm 0.01$ & $0.96 \pm 0.03$ & 537 & $0.45 \pm 0.04$ & 12 \\
$\Sigma_{\mathrm{HI}}$ & $-1.08 \pm 0.03$ & $1.40 \pm 0.05$ & 670 & $0.55 \pm 0.03$ & 17 \\
$\Sigma_{\mathrm{GAS}}$ & $-1.12 \pm 0.03$ & $1.30 \pm 0.05$ & 668 & $0.59 \pm 0.03$ & 19 \\
\hline
\end{tabular}

Notes. Ordinary-least squares fits of the bisector $\log \left(\Sigma_{\mathrm{SFR}}\right)=a_{\mathrm{c}}+$ $b \log (\Sigma)$, where $\Sigma_{\mathrm{SFR}}$ is the face-on surface density of the star formation rate in $M_{\odot} \mathrm{Gyr}^{-1} \mathrm{pc}^{-2}$ and $\Sigma$ the face-on value of the gas surface density in $M_{\odot} \mathrm{pc}^{-2} ; n$ is the number of independent points; $r_{\mathrm{c}}$ is the correlation coefficient and $t$ the student-t test.

value of the total gas surface density in a galaxy. Bigiel et al. (2008) arrived at the same conclusion after comparing the galaxies in their sample.

The correlation between $\Sigma_{\mathrm{SFR}}$ and total gas surface density is slightly better than that between $\Sigma_{\mathrm{SFR}}$ and molecular gas surface density. The bisector fit in Table 7 yields the Kennicutt-Schmidt-law

$\Sigma_{\mathrm{SFR}}=(0.076 \pm 0.005) \Sigma_{\mathrm{GAS}}^{1.30 \pm 0.05}$,

where $\Sigma_{\mathrm{GAS}}$ and $\Sigma_{\mathrm{SFR}}$ are in $M_{\odot} \mathrm{pc}^{-2}$ and $M_{\odot} \mathrm{Gyr}^{-1} \mathrm{pc}^{-2}$, respectively. The exponent of $1.30 \pm 0.05$ is well in the range of 1.1-2.7 derived by Bigiel et al. (2008). As a galaxy of low surface brightness, the SFRs in M 31 are correspondingly low. Our $\Sigma_{\mathrm{SFR}}-\Sigma_{\mathrm{GAS}}$ relationship nicely fits on the low-brightness extension of the compilation of available galaxy data in Fig. 15 of Bigiel et al. (2008), formed by the outer parts of their 7 galaxies and the global values for 20 galaxies of low surface brightness.

Very recently, Braun et al. (2009) also studied the dependence of SFR on gas density in M 31 using the new Westerbork HI survey and the CO survey of Nieten et al. (2006). They estimated the SFR from the surface brightnesses at IRAC $8 \mu \mathrm{m}$, MIPS $24 \mu \mathrm{m}$ and GALEX FUV following the procedure of Thilker et al. (2005). Our Fig. 15a is comparable to the radial range $8-16 \mathrm{kpc}$ in their Fig. 20D that shows the same range in $\Sigma_{\text {SFR }}$ as we find. Note that the molecular gas densities of Braun et al. (2009) are a factor of 1.6 larger (+0.21 dex) and have a wider dynamic range than our values due to differences in scaling of the $\mathrm{CO}$ data, inclination, angular resolution and radial range. Scaling our relationship to the assumptions of Braun et al. (2009) gives $\log \left(\Sigma_{\mathrm{SFR}}\right)=-0.44+0.96 \log \left(\Sigma_{2 \mathrm{H}_{2}}\right)$, which is in good agreement with their Fig. 20D.

The dependencies of SFR surface density on total gas surface density in Fig. 15c and in Fig. 20E of Braun et al. (2009) have the same pear-like shape characterized by a broadening towards lower $\Sigma_{\mathrm{SFR}}$ and a rather sharp cut-off near $\Sigma_{\mathrm{GAS}}=10 M_{\odot} \mathrm{pc}^{-2}$. The cut-off comes from the $\Sigma_{\mathrm{SFR}}-\Sigma_{\mathrm{HI}}$ relation (see Fig. 15b) and occurs at the same value as in the bright galaxies analyzed by Bigiel et al. (2008), who interpreted the lack of higher surface mass densities as a saturation effect. Braun et al. (2009) show that in M 31 this truncation indeed vanishes after correcting the HI data for opacity, which could lead to somewhat steeper slopes in Figs. $15 b$ and c.

\subsection{Radial variations in the Kennicutt-Schmidt law}

In Fig. 16a, we plot the mean values in $0.5 \mathrm{kpc}$-wide rings in the plane of M 31 of $\Sigma_{\mathrm{SFR}}$ against those of $\Sigma_{\mathrm{GAS}}$ from $R=6 \mathrm{kpc}$ to $R=16 \mathrm{kpc}$. The points form a big loop with a horizontal branch for $R=6-8.5 \mathrm{kpc}$ and a maximum $\Sigma_{\mathrm{SFR}}$ in the ring $R=10.5-11.0 \mathrm{kpc}$ (see also Fig. 17). This behavior was already noted by Berkhuijsen (1977) and Tenjes \& Haud (1991), who used the number density of HII regions as tracer of SFR and HI gas, and was recently confirmed by Boissier et al. (2007) from GALEX UV data and total gas. Both Berkhuijsen (1977) and Tenjes \& Haud (1991) showed that the differences between the slopes inside and outside the maximum of the starforming ring is greatly reduced when the increase in the scale height of the gas with increasing radius is taken into account. We calculated the scale height, h, from the scale height of the HI gas given by Eq. (13) of Braun (1991), scaled to $D=780 \mathrm{kpc}$, assumed half this value for that of the $\mathrm{H}_{2}$ gas, and a constant scale height for the ionizing stars. Figure $16 \mathrm{~b}$ shows $\Sigma_{\mathrm{SFR}}$ as a function of gas volume density $n_{\mathrm{GAS}}=N(\mathrm{HI}) / 2 \mathrm{~h}+N\left(2 \mathrm{H}_{2}\right) / \mathrm{h}$. The points have moved towards each other, but the horizontal branch remained and the behavior on the starforming ring has become more complicated.

The variations in slope in Fig. 16 are clear evidence of radial variations in the index of the Kennicutt-Schmidt law. Such variations are not specific to M 31 as they are also seen in some of the galaxies analyzed by Bigiel et al. (2008). In order to quantify the variations, we determined the bisectors in scatter plots for three circular rings: $R=7-9 \mathrm{kpc}, R=9-11 \mathrm{kpc}$ and $R=11-13 \mathrm{kpc}$, covering the horizontal branch, the increasing part inside the maximum and the decreasing part outside the maximum, respectively. The results are given in Table 8 . The index for the star fromation law for surface densities is unity for the 7-9 kpc ring and about 1.6 for the other two rings. Hence, the slope of $b=1.30 \pm 0.05$ obtained for the "10 kpc ring" $(R=6.8-$ $11.4 \mathrm{kpc}$ ) in Sect. 7.1 represents the mean value of the first two rings considered here. The scatter plots between $\Sigma_{\mathrm{SFR}}$ and gas volume density yield bisector slopes that are about 0.2 smaller than those for surface density. The correlation coefficients are all close to $r_{\mathrm{c}}=0.59 \pm 0.03$ for the " $10 \mathrm{kpc}$ ring" (see Table 7 ), indicating that even in $2 \mathrm{kpc}$-wide rings the intrinsic scatter is considerable. This implies that on scales of a few hundred parsec significant variations in the index of the Kennicutt-Schmidt law and in the star formation efficiency occur.

\subsection{Radial variations in SFR and SFE}

In Fig. 17 (upper panel) we present the radial profile of the SFR surface density between $6 \mathrm{kpc}$ and $17 \mathrm{kpc}$, averaged in $0.5 \mathrm{kpc}-$ wide circular rings in the plane of M 31. The face-on values vary between about 0.1 and $1 M_{\odot} \mathrm{Gyr}^{-1} \mathrm{pc}^{-2}$. Boissier et al. (2007) and Braun et al. (2009) obtained similar values for $\Sigma_{\text {SFR }}$ in this radial range from GALEX UV data. They are about 10 times smaller than the surface densities of SFR between $R=1.5 \mathrm{kpc}$ and $R=7 \mathrm{kpc}$ in the northern part of M 33 observed by (Gardan et al. 2007).

In the lower panel of Fig. 17 we show the radial profiles of the surface density of the molecular gas and of the star formation efficiencies SFE $=\Sigma_{\mathrm{SFR}} / \Sigma_{2 \mathrm{H}_{2}}$ and $\Sigma_{\mathrm{SFR}} / \Sigma_{\mathrm{GAS}}$. Although the maximum $\Sigma_{\mathrm{SFR}}$ occurs on a relative maximum in the molecular gas density (in ring 10.5-11.0 kpc), $\Sigma_{\text {SFR }}$ is only about $70 \%$ of its maximum value where the molecular gas density is highest (in ring 9.0-9.5 kpc). Consequently, SFE varies significantly with radius. Between $R=6 \mathrm{kpc}$ and $R=15 \mathrm{kpc}$ SFE fluctuates around a value of $0.9 \mathrm{Gyr}^{-1}$, with a minimum of $0.46 \pm 0.01 \mathrm{Gyr}^{-1}$ near $R=9 \mathrm{kpc}$. Thus SFE is smallest where $\Sigma_{2 \mathrm{H}_{2}}$ is highest! Up to $R=12 \mathrm{kpc}$ the efficiency $\Sigma_{\mathrm{SFR}} / \Sigma_{\mathrm{GAS}}$ shows the same trend as SFE. The increase in SFE between $12 \mathrm{kpc}$ and $15 \mathrm{kpc}$ radius of a factor 1.5 results from the difference in radial scale lengths of $\Sigma_{\mathrm{SFR}}$ (or $\mathrm{H} \alpha$ emission) and the molecular gas density (see Fig. 6 and Table 3). Interestingly, in M 33 (Gardan et al. 2007) found a radial increase in SFE of a factor 2 between 


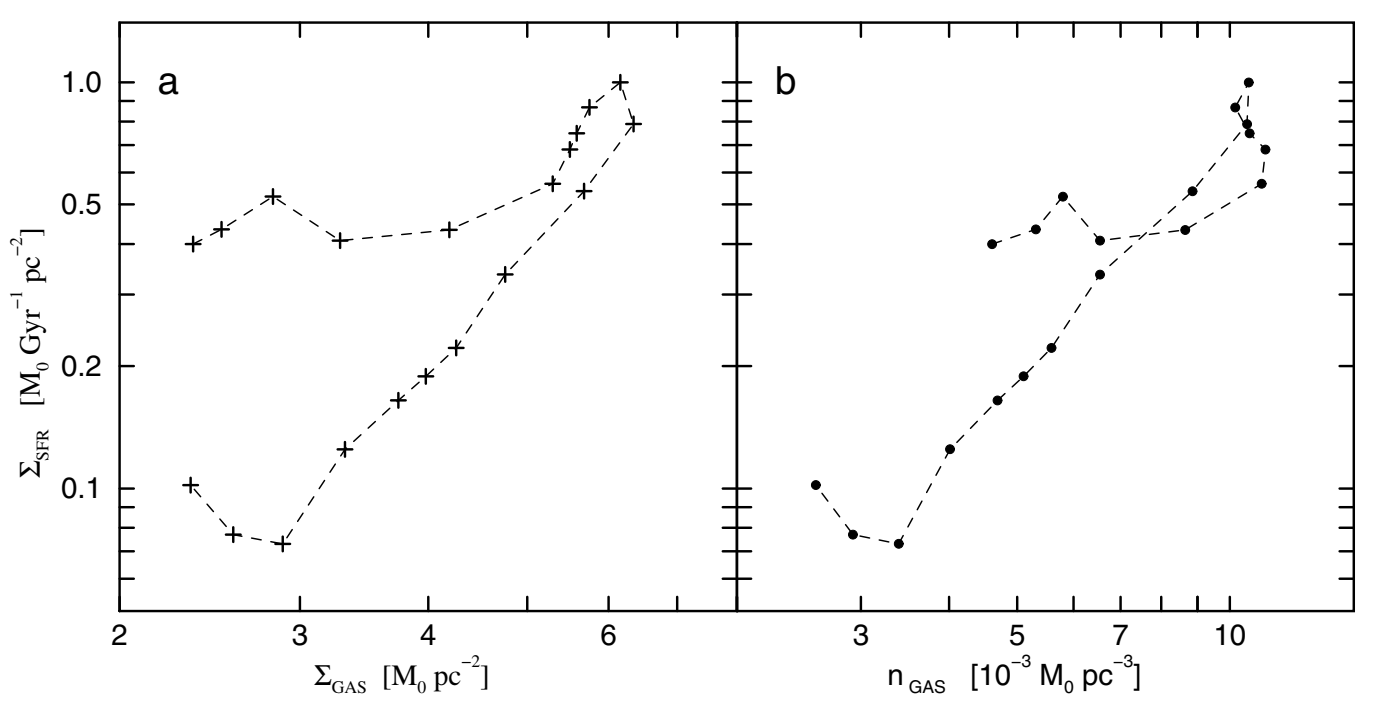

Fig. 16. Mean face-on values of $\Sigma_{\mathrm{SFR}}$, averaged in $0.5 \mathrm{kpc}$-wide circular rings in the plane of M 31, plotted against the corresponding mean values of a) gas surface density $\Sigma_{\mathrm{GAS}}$, and b) gas volume density $n_{\mathrm{GAS}}$. The upper left point is for the ring $R=6.0-6.5 \mathrm{kpc}$, the maximum in $\Sigma_{\mathrm{SFR}}$ is in ring $10.5-11.0 \mathrm{kpc}$ and the minimum in ring $R=14.5-15.0 \mathrm{kpc}$. Typical errors are $0.01 M_{\odot} \mathrm{Gyr}^{-1} \mathrm{pc}^{-2}$ in $\Sigma_{\mathrm{SFR}}, 0.02 M_{\odot} \mathrm{pc}^{-2}$ in $\Sigma_{\mathrm{GAS}}$, and $3 \times 10^{-5} M_{\odot} \mathrm{pc}^{-3}$ in $n_{\mathrm{GAS}}$. Points for $R>12 \mathrm{kpc}$ suffer from missing data points near the major axis, the number of which increases with radius.

Table 8. Kennicutt-Schmidt law in three radial intervals in M 31.

\begin{tabular}{lccclcl}
\hline \hline \multicolumn{1}{c}{$R(\mathrm{kpc})$} & $\mathrm{X}$ & $a_{\mathrm{c}}$ & $b$ & $n$ & $r_{\mathrm{c}}$ & $t$ \\
\hline $7-9$ & $\Sigma_{\mathrm{GAS}}$ & $-0.90 \pm 0.04$ & $1.03 \pm 0.06$ & 216 & $0.54 \pm 0.06$ & 9 \\
$9-11$ & & $-1.43 \pm 0.06$ & $1.67 \pm 0.08$ & 356 & $0.63 \pm 0.04$ & 15 \\
$11-13$ & & $-1.46 \pm 0.06$ & $1.55 \pm 0.08$ & 297 & $0.62 \pm 0.05$ & 13 \\
& & & & & & \\
$7-9$ & $n_{\mathrm{GAS}}$ & $0.18 \pm 0.04$ & $0.88 \pm 0.06$ & 218 & $0.51 \pm 0.06$ & 9 \\
$9-11$ & & $0.45 \pm 0.04$ & $1.50 \pm 0.07$ & 356 & $0.62 \pm 0.04$ & 15 \\
$11-13$ & & $0.35 \pm 0.04$ & $1.35 \pm 0.07$ & 297 & $0.62 \pm 0.05$ & 14 \\
\hline
\end{tabular}

Notes. Ordinary least-squares fits of the bisector $\log \left(\Sigma_{\mathrm{SFR}}\right)=a_{\mathrm{c}}+b \log (X)$, where $\Sigma_{\mathrm{SFR}}$ is the face-on surface density of the star formation rate in $M_{\odot} \mathrm{Gyr}^{-1} \mathrm{pc}^{-2}, X=\Sigma_{\mathrm{GAS}}$ is the face-on gas surface density in $M_{\odot} \mathrm{pc}^{-2}$ and $X=n_{\mathrm{GAS}}$ is the gas volume density in at $\mathrm{cm}^{-3} . n$ is the number of independent points; $r_{\mathrm{c}}$ is the correlation coefficient and $\mathrm{t}$ the student- $\mathrm{t}$ test.

$2 \mathrm{kpc}$ and $6 \mathrm{kpc}$ radius with similar fluctuations around the mean as we observe in M 31, but the mean value in M 31 is about three times lower than in M 33. Furthermore, Leroy et al. (2008) found significant variations in the efficiency $\Sigma_{\mathrm{SFR}} / \Sigma_{\mathrm{GAS}}$ on a linear scale of $800 \mathrm{pc}$ in the sample of 12 spiral galaxies analyzed by them.

That large, small-scale variations in SFE exist in galaxies is also clear from the large spread in the scatter plots of $\Sigma_{\mathrm{SFR}}-\Sigma_{2 \mathrm{H}_{2}}$ visible in Fig. 15a and in several figures of Bigiel et al. (2008). The same value of $\Sigma_{\mathrm{SFR}}$ can occur in a range of $\Sigma_{2 \mathrm{H}_{2}}$ spanning more than a factor of 10 .

We may conclude that neither the present-day star formation rate $\Sigma_{\mathrm{SFR}}$ nor the star formation efficiency SFE is well correlated with the molecular gas surface density. Hence, other factors than molecular gas density must play an important role in the star formation process. Bigiel et al. (2008) argue that local environmental circumstances largely determine the SFE in spiral galaxies. These factors are extensively discussed by e.g. Leroy et al. (2008).

\section{Summary}

In this paper, we studied the emission from dust, neutral gas and ionized gas in the disk of $\mathrm{M} \mathrm{31}$, and the relationships between these components on various linear scales. We compared the Spitzer MIPS maps at $24 \mu \mathrm{m}, 70 \mu \mathrm{m}$ and $160 \mu \mathrm{m}$ (Gordon et al. 2006) to the distributions of atomic gas seen in the HI line (Brinks \& Shane 1984), molecular gas as traced by the ${ }^{12} \mathrm{CO}(1-0)$ line (Nieten et al. 2006) and ionized gas observed in $\mathrm{H} \alpha$ (Devereux et al. 1994). All data were smoothed to an angular resolution of $45^{\prime \prime}$ corresponding to $170 \mathrm{pc} \times 660 \mathrm{pc}$ in the plane of the galaxy.

For each of the dust and gas maps, we calculated the mean intensity distribution as a function of radius (Fig. 6), separately for the northern and the southern half of M 31. Using wavelet analysis, we decomposed the dust and gas distributions in spatial scales and calculated cross-correlations as a function of scale. We also used classical correlations to derive quantitative relations between the various dust and gas components.

Using the MIPS $70 \mu \mathrm{m}$ and $160 \mu \mathrm{m}$ maps, we derived the distributions of the dust temperature and optical depth. The dust optical depth at the $\mathrm{H} \alpha$ wavelength was used to a) investigate the dust-to-gas ratio, b) derive scaling relations between extinction and neutral gas emission, and c) de-redden the $\mathrm{H} \alpha$ emission in order to estimate the recent star formation rate. We also presented the Kennicutt-Schmidt law indices obtained for the bright emission ring near $R=10 \mathrm{kpc}$ in M 31 . We summarize the main results and conclusions as follows. 


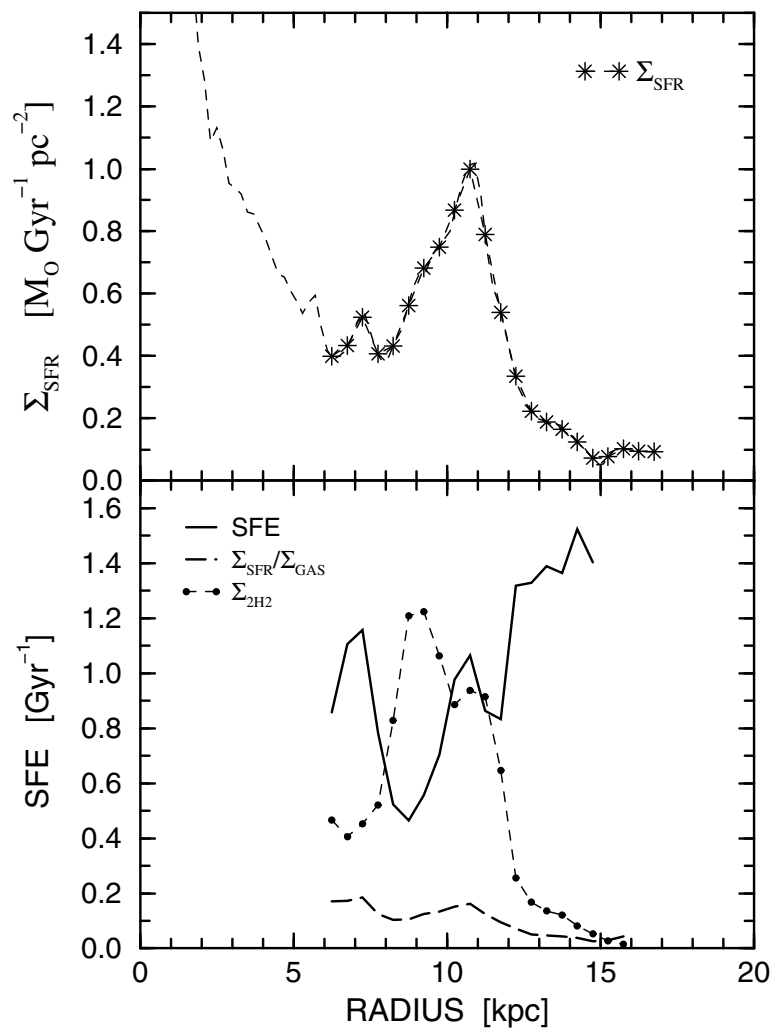

Fig. 17. Radial variation of the face-on surface density of the star formation rate $\Sigma_{\text {SFR }}$ and star formation efficiency SFE in M 31, averaged in $0.5 \mathrm{kpc}$-wide rings in the plane of the galaxy. Beyond $R=12 \mathrm{kpc}$ the data are not complete, because the observed area is limited along the major axis (see Fig. 10a). Top: radial profile of $\Sigma_{\mathrm{SFR}}$. Bottom: full line - SFE $=\Sigma_{\mathrm{SFR}} / \Sigma_{2 \mathrm{H}_{2}}$; long dashed line $-\Sigma_{\mathrm{SFR}} / \Sigma_{\mathrm{GAS}}$; dots - radial profile of the molecular gas surface density $\Sigma_{2 \mathrm{H}_{2}}$ seen face-on. Note that $\Sigma_{\mathrm{SFR}}$ and $\Sigma_{2 \mathrm{H}_{2}}$ do not peak at the same radius. Statistical errors in $\Sigma_{\mathrm{SFR}}$ and $\Sigma_{2 \mathrm{H}_{2}}$ are smaller than the symbols and those in SFE and $\Sigma_{\mathrm{SFR}} / \Sigma_{\mathrm{GAS}}$ are smaller than the thickness of the lines. Only beyond $R=12 \mathrm{kpc}$ the error in SFE slowly increases to $0.3 \mathrm{Gyr}^{-1}$ at $R=15 \mathrm{kpc}$.

\section{Dust temperature and opacity:}

- The dust temperature steeply drops from about $30 \mathrm{~K}$ in the center to about $19 \mathrm{~K}$ near $R=4.5 \mathrm{kpc}$, and stays between about $17 \mathrm{~K}$ and $20 \mathrm{~K}$ beyond this radius (Fig. 2). The mean dust temperature in the area studied is about $18.5 \mathrm{~K}$. This is $3 \mathrm{~K}$ less than the temperature obtained by Walterbos \& Schwering (1987) between the IRAS maps at $60 \mu \mathrm{m}$ and $100 \mu \mathrm{m}$ that both trace warmer dust than the MIPS maps at $70 \mu \mathrm{m}$ and $160 \mu \mathrm{m}$ used here.

- The dust optical depth at $\mathrm{H} \alpha$ along the line of sight varies in a range between about 0.2 near the center and about 1 in the "10 kpc ring" (Fig. 4) with a mean value of $0.7 \pm 0.4$ (the error is standard deviation) and a most probable value of $\simeq 0.5$, indicating that M 31 is mostly optically thin to the $\mathrm{H} \alpha$ emission. The total flux density of the $\mathrm{H} \alpha$ emission increases by $30 \%$ after correction for extinction.

\section{Radial distributions:}

- The radial scale lengths between the maximum in the " $10 \mathrm{kpc}$ ring" and $R=15 \mathrm{kpc}$ of the warm dust are smaller than that of the cold dust, as is expected if the warm dust is mainly heated by UV photons from star forming regions and cold dust by the
ISRF. With the largest scale length, atomic gas has the largest radial extent of the dust and gas components considered here.

- The radial gradient of the total gas-to-dust ratio is consistent with that of the oxygen abundance in M 31. The gas-to-dust ratios observed in the solar neighborhood (Bohlin et al. 1978) occur near $R=8.5 \mathrm{kpc}$ in the disk of M 31 where $N($ gas $) / \tau_{\mathrm{H} \alpha}=2.6 \times 10^{21}$ at $\mathrm{cm}^{-2}$.

\section{Properties as a function of scale:}

- Spatial scales larger than about $8 \mathrm{kpc}$ contain most of the emitted power from the cold dust and the atomic gas, whereas the emissions from warm dust, molecular gas and ionized gas are dominated by scales near $1 \mathrm{kpc}$, typical for complexes of starforming regions and molecular clouds in spiral arms (Fig. 11).

- Dust emission is correlated $\left(r_{w} \geq 0.6\right)$ with both neutral and ionized gas on scales $>1 \mathrm{kpc}$.

- On scales $<1 \mathrm{kpc}$, ionized gas is best correlated with warm dust and neutral gas (both $\mathrm{HI}$ and $\mathrm{H}_{2}$ ) with cold dust. On the smallest scale of $0.4 \mathrm{kpc}$, an HI-warm dust correlation hardly exists $\left(r_{w} \simeq 0.4\right)$ because not much HI occurs on the scale of starforming regions (see Fig. 11).

\section{Relationships between gas and dust:}

- $\mathrm{H} \alpha$ emission is slightly better correlated with the emission at $70 \mu \mathrm{m}$ than at $24 \mu \mathrm{m}$ (Fig. 13, Table 6), especially on scales $<2 \mathrm{kpc}$ (Fig. 12). As in M 33 the $24 \mu \mathrm{m}-\mathrm{H} \alpha$ correlation is best, this suggests that in early-type galaxies like M 31 the contribution from evolved AGB stars to the $24 \mu \mathrm{m}$ emission is larger than in late-type galaxies like M 33.

- Dust extinction $A_{H \alpha}$ is not well correlated with $N\left(2 \mathrm{H}_{2}\right)$ indicating that dust mixed with molecular clouds does not contribute much to the total extinction. Although the correlation with $N(\mathrm{HI})$ is better, $A_{H \alpha}$ is best correlated with $N\left(\mathrm{HI}+2 \mathrm{H}_{2}\right)$.

- Dust opacity is proportional to the square root of $N\left(2 \mathrm{H}_{2}\right)$ but about linearly related to $N(\mathrm{HI})$, as was also found by Nieten et al. (2006) at 90" resolution. This is an indirect indication of a balance between the formation and destruction rates of $\mathrm{H}_{2}$ in cool, dusty HI clouds.

- In the central $2 \mathrm{kpc}$ both the dust opacity and the HI column density are very low and the dust temperature is high. This combination may explain the lack of $\mathrm{H}_{2}$ in this region.

\section{SFR and SFE:}

- The SFR in M 31 is low. The total SFR in the observed field between $R=6 \mathrm{kpc}$ and $R=17 \mathrm{kpc}$ is $0.27 M_{\odot} \mathrm{yr}^{-1}$ and the star formation efficiency is $0.9 \mathrm{Gyr}^{-1}$, yielding a molecular depletion time scale of $1.1 \mathrm{Gyr}$. This is about three times longer than observed in the northern part of M 33 (Gardan et al. 2007). The radial distribution of $\Sigma_{\mathrm{SFR}}$ in $0.5 \mathrm{kpc}$-wide rings in the plane of the galaxy (Fig. 17) varies between about 0.1 and $1 M_{\odot} \mathrm{Gyr}^{-1} \mathrm{pc}^{-2}$, values that are about 10 times smaller than in the northern part of M 33 (Gardan et al. 2007). Between $R=6 \mathrm{kpc}$ and $R=15 \mathrm{kpc}$, SFE varies between about $0.5 \mathrm{Gyr}^{-1}$ and $1.5 \mathrm{Gyr}^{-1}$, whereas the efficiency with respect to the total gas surface density slowly decreases from about $0.18 \mathrm{Gyr}^{-1}$ to about $0.03 \mathrm{Gyr}^{-1}$.

- SFR is not well correlated with neutral gas and worst of all with molecular gas in the radial range $30^{\prime}-50^{\prime}$ containing the "10 kpc ring" (Fig. 15, Table 7). In spite of this, the 
power-law fits are statistically significant. We find a linear relationship between the surface densities of SFR and molecular gas (power-law exponent $0.96 \pm 0.03$ ), and a power law with index $1.30 \pm 0.05$ between the surface densities of SFR and total gas. These results agree with the average relationship for 7 nearby galaxies much brighter than M 31 (Bigiel et al. 2008). While in these galaxies molecular hydrogen is the dominant gas phase, most of the neutral gas in M 31 is atomic. Thus, the surface density of SFR depends linearly on that of molecular gas irrespective of the fraction of molecular gas or the absolute value of the total gas surface density in a galaxy.

\section{Some important implications of this study are:}

- Precaution is required in using the total IR luminosity (TIR) as an indicator of recent SFR or to derive dust opacity for an early-type galaxy like M 31, because the cold dust is mainly heated by the ISRF and the warm dust emission at $24 \mu \mathrm{m}$ is partly due to evolved stars (especially in the bulge of the galaxy).

- Neither the present-day SFR nor SFE is well correlated with the surface density of molecular gas or total gas. Therefore, other factors than gas density must play an important role in the process of star formation in M 31.

Acknowledgements. We are grateful to E. Krügel for valuable and stimulating comments. We thank K.M. Menten and R. Beck for comments and careful reading of the manuscript. The Spitzer MIPS data were kindly provided by Karl D. Gordon. E. Tempel kindly sent us a table of extinction values that we used for Fig. 5. We thank an anonymous referee for extensive comments leading to improvements in the manuscript. F.T. was supported through a stipend from the Max Planck Institute for Radio Astronomy (MPIfR).

\section{References}

Andriesse, C. D. 1974, A\&A, 37, 257

Bajaja, E., \& Gergely, T. E. 1977, A\&A, 61, 229

Barmby, P., Ashby, M. L. N., Bianchi, L., et al. 2006, ApJ, 650, L45

Berkhuijsen, E. M. 1977, A\&A, 57, 9

Berkhuijsen, E. M., \& Humphreys, R. M. 1989, A\&A, 214, 68

Bigiel, F., Leroy, A., Walter, F., et al. 2008, AJ, 136, 2846

Blair, W. P., Kirshner, R. P., \& Chevalier, R. A. 1982, ApJ, 254, 50

Block, D. L., Bournaud, F., Combes, F., et al. 2006, Nature, 443, 832

Bohlin, R. C., Savage, B. D., \& Drake, J. F. 1978, ApJ, 224, 132

Boissier, S., Gil de Paz, A., Boselli, A., et al. 2007, ApJS, 173, 524

Boulanger, F., Abergel, A., Bernard, J.-P., et al. 1996, A\&A, 312, 256

Braun, R. 1990, ApJS, 72, 755

Braun, R. 1991, ApJ, 372, 54

Braun, R., Thilker, D. A., Walterbos, R. A. M., \& Corbelli, E. 2009, ApJ, 695, 937

Brinks, E., \& Shane, W. W. 1984, A\&AS, 55, 179

Calzetti, D., Kennicutt, Jr., R. C., Bianchi, L., et al. 2005, ApJ, 633, 871

Calzetti, D., Kennicutt, R. C., Engelbracht, C. W., et al. 2007, ApJ, 666, 870

Chemin, L., Carignan, C., \& Foster, T. 2009, ApJ, 705, 1395

Ciardullo, R., Rubin, V. C., Ford, Jr., W. K., Jacoby, G. H., \& Ford, H. C. 1988, AJ, 95, 438

Cox, P., Kruegel, E., \& Mezger, P. G. 1986, A\&A, 155, 380

Dennefeld, M., \& Kunth, D. 1981, AJ, 86, 989

Devereux, N. A., Price, R., Wells, L. A., \& Duric, N. 1994, AJ, 108, 1667

Dickinson, C., Davies, R. D., \& Davis, R. J. 2003, MNRAS, 341, 369

Diplas, A., \& Savage, B. D. 1994, ApJ, 427, 274

Draine, B. T., \& Lee, H. M. 1984, ApJ, 285, 89

Draine, B. T., Dale, D. A., Bendo, G., et al. 2007, ApJ, 663, 866

Emerson, D. T. 1974, MNRAS, 169, 607
Evans, I. N. 1986, ApJ, 309, 544

Frick, P., Beck, R., Berkhuijsen, E. M., \& Patrickeyev, I. 2001, MNRAS, 327, 1145

Gardan, E., Braine, J., Schuster, K. F., Brouillet, N., \& Sievers, A. 2007, A\&A, 473, 91

Garnett, D. R., Shields, G. A., Skillman, E. D., Sagan, S. P., \& Dufour, R. J. 1997, ApJ, 489, 63

Gordon, K. D., Rieke, G. H., Engelbracht, C. W., et al. 2005, PASP, 117, 503

Gordon, K. D., Bailin, J., Engelbracht, C. W., et al. 2006, ApJ, 638, L87

Gordon, K. D., Engelbracht, C. W., Fadda, D., et al. 2007, PASP, 119, 1019

Haas, M., Lemke, D., Stickel, M., et al. 1998, A\&A, 338, L33

Henry, R. B. C., \& Howard, J. W. 1995, ApJ, 438, 170

Hippelein, H., Haas, M., Tuffs, R. J., et al. 2003, A\&A, 407, 137

Hirashita, H. 1999, ApJ, 510, L99

Hirashita, H., Tajiri, Y. Y., \& Kamaya, H. 2002, A\&A, 388, 439

Hoernes, P., Berkhuijsen, E. M., \& Xu, C. 1998, A\&A, 334, 57

Isobe, T., Feigelson, E. D., Akritas, M. G., \& Babu, G. J. 1990, ApJ, 364, 104

Issa, M. R., MacLaren, I., \& Wolfendale, A. W. 1990, A\&A, 236, 237

Kang, Y., Bianchi, L., \& Rey, S. 2009, ApJ, 703, 614

Kennicutt, Jr., R. C. 1998a, ARA\&A, 36, 189

Kennicutt, Jr., R. C. 1998b, ApJ, 498, 541

Kennicutt, Jr., R. C., Calzetti, D., Walter, F., et al. 2007, ApJ, 671, 333

Kennicutt, R. C., Hao, C., Calzetti, D., et al. 2009, ApJ, 703, 1672

Krügel, E. 2003, The physics of interstellar dust, ed. E. Krügel, IoP Series in Astronomy and Astrophysics (Bristol, UK: The Institute of Physics)

Krügel, E. 2009, A\&A, 493, 385

Leroy, A. K., Walter, F., Brinks, E., et al. 2008, AJ, 136, 2782

Magnier, E. A., Hodge, P., Battinelli, P., Lewin, W. H. G., \& van Paradijs, J. 1997, MNRAS, 292, 490

Montalto, M., Seitz, S., Riffeser, A., et al. 2009, A\&A, 507, 283

Nakai, N., \& Sofue, Y. 1982, PASJ, 34, 199

Nakai, N., \& Sofue, Y. 1984, PASJ, 36, 313

Nedialkov, P., Berkhuijsen, E. M., Nieten, C., \& Haas, M. 2000, in Proceedings, WE-Heraeus Seminar, ed. E. M. Berkhuijsen, R. Beck, \& R. A. M. Walterbos, 85,232

Neininger, N., Guélin, M., Ungerechts, H., Lucas, R., \& Wielebinski, R. 1998, Nature, 395, 871

Nieten, C., Neininger, N., Guélin, M., et al. 2006, A\&A, 453, 459

Pagel, B. E. J., \& Edmunds, M. G. 1981, ARA\&A, 19, 77

Pagel, B. E. J., Edmunds, M. G., Blackwell, D. E., Chun, M. S., \& Smith, G. 1979, MNRAS, 189, 95

Pérez-González, P. G., Kennicutt, Jr., R. C., Gordon, K. D., et al. 2006, ApJ, 648, 987

Reach, W. T., \& Boulanger, F. 1998, in The Local Bubble and Beyond, Proc. IAU Colloq. 166, ed. D. Breitschwerdt, M. J. Freyberg, \& J. Truemper (Berlin: Springer Verlag), Lect. Notes Phys., 506, 353

Relaño, M., Lisenfeld, U., Pérez-González, P. G., Vílchez, J. M., \& Battaner, E. 2007, ApJ, 667, L141

Rieke, G. H., Young, E. T., Engelbracht, C. W., et al. 2004, ApJS, 154, 25

Savage, B. D., Wesselius, P. R., Swings, J. P., \& The, P. S. 1978, ApJ, 224, 149

Savcheva, A. S., \& Tassev, S. V. 2002, Publications de l'Observatoire Astronomique de Beograd, 73, 219

Schmidt, M. 1959, ApJ, 129, 243

Soifer, B. T., Rice, W. L., Mould, J. R., et al. 1986, ApJ, 304, 651

Stanek, K. Z., \& Garnavich, P. M. 1998, ApJ, 503, L131

Tabatabaei, F. S., Beck, R., Krause, M., et al. 2007a, A\&A, 466, 509

Tabatabaei, F. S., Beck, R., Krügel, E., et al. 2007b, A\&A, 475, 133

Tempel, E., Tamm, A., \& Tenjes, P. 2010, A\&A, 509, 91

Tenjes, P., \& Haud, U. 1991, A\&A, 251, 11

Thilker, D. A., Hoopes, C. G., Bianchi, L., et al. 2005, ApJ, 619, L67

Trundle, C., Dufton, P. L., Lennon, D. J., Smartt, S. J., \& Urbaneja, M. A. 2002, A\&A, 395, 519

Unwin, S. C. 1980, MNRAS, 192, 243

van Genderen, A. M. 1973, A\&A, 24, 47

Verley, S., Corbelli, E., Giovanardi, C., \& Hunt, L. K. 2009, A\&A, 493, 453

Viallefond, F., Goss, W. M., \& Allen, R. J. 1982, A\&A, 115, 373

Walterbos, R. A. M., \& Braun, R. 1994, ApJ, 431, 156

Walterbos, R. A. M., \& Kennicutt, Jr., R. C. 1988, A\&A, 198, 61

Walterbos, R. A. M., \& Schwering, P. B. W. 1987, A\&A, 180, 27

Williams, B. F. 2003, AJ, 126, 1312

Witt, A. N., \& Gordon, K. D. 2000, ApJ, 528, 799

Xu, C., \& Helou, G. 1996, ApJ, 456, 163

Yin, J., Hou, J. L., Prantzos, N., et al. 2009, A\&A, 505, 497 